\title{
Non Fermi Liquid Ground States in Strongly Correlated f-Electron Materials
}

\author{
M. B. Maple, $§$ M. C. de Andrade, $\$$ J. Herrmann,, Y. Dalichaouch,, \\ D. A. Gajewski, $₫$ C. L. Seaman, $₫$ R. Chau,, \\ R. Movshovich, ${ }^{*}$ M. C. Aronson, ${ }^{\dagger}$ and R. Osborn $\ddagger$ \\ $\S_{\text {Department }}$ Physics and Institute for Pure and Applied Physical Sciences, \\ University of California, San Diego, La Jolla, CA 92093-0319 \\ * Los Alamos National Laboratory, Los Alamos, NM 87545 \\ †The Harrison M. Randall Laboratory of Physics, \\ University of Michigan, Ann Arbor, MI 48109-1120

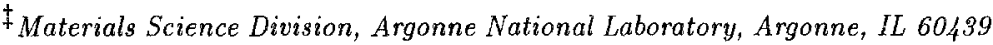

Experimental efforts to characterize and develop an understanding of non Fermi liquid (NFL) behavior at low temperature in f-electron materials are reviewed for three f-electron systems: $M_{1-x} U_{x} P d_{3} \quad(M=S c, Y), U_{1-x} T h_{x} P d_{2} A l_{3}$, and $U C u_{5-x} P d_{x}$. The emerging systematics of NFL behavior in f-electron systems, based on the present sample of nearly ten f-electron systems, is updated. Many of the f-electron systems exhibit the following temperature dependences of the electrical resistivity $\rho$, specific heat $C$, and magnetic susceptibility $\chi$ for $T \ll T_{0}$, where $T_{0}$ is a characteristic temperature: $\rho(T) \sim 1-a T / T_{0}$, where $a<0$ or $>0$, $C(T) / T \sim\left(-1 / T_{0}\right) \ln \left(T / b T_{0}\right)$, and $\chi(T) \sim 1-c\left(T / T_{0}\right)^{1 / 2}$. In several of the $f$ electron systems, the characteristic temperature $T_{0}$ can be identified with the Kondo temperature $T_{K}$.

\section{INTRODUCTION}

During the past several years, there has been a great deal of interest in a new class of f-electron heavy fermion materials which exhibit non Fermi liquid (NFL) behavior at low temperatures. These materials are $\mathrm{Ce}$ and $\mathrm{U}$ intermetallics which, with a few possible exceptions, have been doped with a nonmagnetic element. The $\mathrm{Ce}$ and $\mathrm{U}$ ions have partially-filled f-electron shells and contribute magnetic dipole or electric quadrupole moments which can interact with the spins and charges of the conduction electrons and participate in magnetic and quadrupole ordering at low temperatures. What is striking about these materials is the non Fermi liquid behavior of their physical properties which exhibit weak power law or logarithmic divergences in temperature and suggest the existence of a critical point at $T=$ 
$0 \mathrm{~K}$. One possible origin of the $0 \mathrm{~K}$ critical point is an unconventional moment compensation process such as a multichannel Kondo effect. Such a single ion effect would seem to be appropriate for systems such as $\mathrm{Y}_{1-x} \mathrm{U}_{x} \mathrm{Pd}_{3}, \mathrm{U}_{1-x} \mathrm{Th}_{x} \mathrm{Pd}_{2} \mathrm{Al}_{3}$, and $\mathrm{La}_{1-x} \mathrm{Ce}_{x} \mathrm{Cu}_{2} \mathrm{Si}_{2}$, where the NFL characteristics persist to low concentrations of $\mathrm{Ce}$ or $\mathrm{U}$. Another candidate for the source of the $0 \mathrm{~K}$ critical point is fluctuations of the order parameter in the vicinity of a $0 \mathrm{~K}$ second order phase transition. Such a transition could be associated with long-range or glassy magnetic or quadrupolar order. Systems in which this seems to be a viable mechanism include $\mathrm{UCu}_{3.5} \mathrm{Pd}_{1.5}$ and $\mathrm{CeCu}_{5.9} \mathrm{Au}_{0.1}$, in which the $\mathrm{Ce}$ and $\mathrm{U}$ sublattices are atomically ordered.

Some of the interest in non Fermi liquid behavior in strongly correlated electron systems, particularly copper oxides and f-electron materials, is associated with the unconventional superconductivity found in these two classes of materials. In spite of the disparity in the values of the superconducting critical temperatures $T_{\varepsilon}$, which are as high as $\sim 133 \mathrm{~K}$ for the copper oxide superconductors but only $\leqslant 2 \mathrm{~K}$ for the f-electron heavy fermion materials, the superconducting states of both of these classes of materials share some striking similarities - the superconducting state appears to be anisotropic, with an energy gap that may vanish at points or lines on the Fermi surface, and the superconducting electron pairing may be mediated by antiferromagnetic spin fluctuations. An understanding of the source of the NFL behavior in these systems may provide important information about the electronic structure and excitations in these systems, as well as the origin of the unconventional superconductivity.

In this paper, we describe recent experimental efforts to characterize and develop an understanding of NFL behavior at low temperature in f-electron materials. Experiments are reviewed for three f-electron systems: $\mathrm{M}_{1-x} \mathrm{U}_{x} \mathrm{Pd} \mathrm{d}_{3}(\mathrm{M}=\mathrm{Sc}, \mathrm{Y})$, $\mathrm{U}_{1-x} \mathrm{Th}_{x} \mathrm{Pd}_{2} \mathrm{Al}_{3}$, and $\mathrm{UCu}_{5-x} \mathrm{Pd}_{x}$. We also give an update of the emerging systematics of NFL behavior in f-electron systems, based on the present sample of nearly ten f-electron systems.

\section{THE $\mathrm{M}_{1-x} \mathrm{U}_{x} \mathrm{Pd}_{3}(\mathrm{M}=\mathrm{Sc}, \mathrm{Y})$ SYSTEM}

The $\mathrm{M}_{1-x} \mathrm{U}_{x} \mathrm{Pd}_{3}(\mathrm{M}=\mathrm{Sc}, \mathrm{Y}$, lanthanide) system exhibits many interesting phenomena including a structural phase transition, spin-glass freezing, crystalline electric field (CEF) effects, Fermi level tuning, and unusual Kondo behavior. ${ }^{1,2}$ of particular interest is the Kondo behavior, which occurs at low $U$ concentrations for $\mathrm{M}=\mathrm{Sc}$ and $\mathrm{Y}$ and has low temperature properties with NFL characteristics.

Shown in Fig. 1 is the temperature - U concentration $(T-x)$ phase diagram of $\mathrm{Y}_{1-x} \mathrm{U}_{x} \mathrm{Pd}_{3}$ which summarizes the general behavior as a function of $x$. There is a mixed phase region for $0.6 \leq x \leq 0.8$. The samples which form in the hexagonal $\mathrm{Ni}_{3} \mathrm{Ti}$ crystal structure $(0.9 \leq x \leq 1)$ appear to have nonmagnetic ground states, although $\mathrm{UPd}_{3}$ has recently been studied by inelastic neutron scattering and reported to undergo a quadrupolar transition at $6.5 \mathrm{~K}$, followed by a magnetic transition at $4.5 \mathrm{~K}^{3}$ The samples which form in the cubic Cu${ }_{3} \mathrm{Au}$ structure $(0 \leq x \leq 0.5)$ with higher $U$ concentrations $(0.3 \leqslant x \leqslant 0.5)$ exhibit spin-glass freezing below a temperature $T_{S G}$ which increases monotonically with $x$, as shown in Fig. 1. Such an increase is expected for RKKY interactions whose strength increases as $\sim \mathcal{J}^{2} \mathcal{N}\left(E_{F}\right) x$ where $\mathcal{J}$ is the exchange interaction parameter and $\mathcal{N}\left(E_{F}\right)$ is the density of states at the 


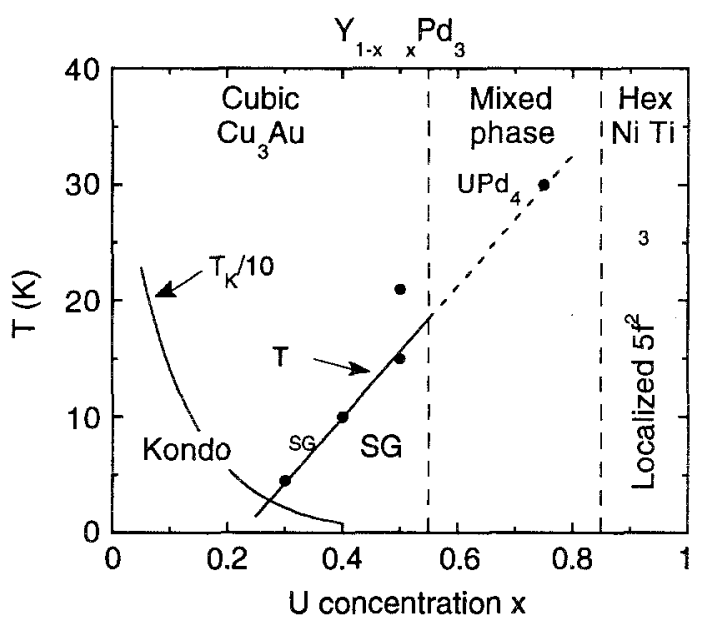

Fig. 1. Low temperature - $\mathrm{U}$ concentration phase diagram of $\mathrm{Y}_{1-x} \mathrm{U}_{x} \mathrm{Pd}_{3}$. From Ref. 2 .

Fermi level. Recent muon spin relaxation measurements on $\mathrm{Y}_{1-x} \mathrm{U}_{x} \mathrm{Pd}_{3}$ demonstrate that the spin-glass order in this system can be understood in the context of induced moments. ${ }^{4}$

For $0<x \leq 0.2, \mathrm{Y}_{1-x} \mathrm{U}_{x} \mathrm{Pd}_{3}$ exhibits unusual Kondo behavior with a Kondo temperature $T_{K}$ that increases with decreasing $x$, as indicated in Fig. 1. This is consistent with the "Fermi level tuning" revealed by previous photoemission studies of $\mathrm{Y}_{1-x} \mathrm{U}_{x} \mathrm{Pd}_{3}$, where it was found that the separation between $E_{F}$ and the $5 \mathrm{f}$ peak below it, $\left|E_{F}-E_{5 f}\right|$, decreases with decreasing $x$ as $\mathrm{UPd}_{3}$ is diluted with Y. ${ }^{5}$ This was interpreted as "Fermi level tuning" because substitution of trivalent $Y$ for tetravalent $U$ causes a decrease in the conduction electron density and, in turn, $E_{F}$. Because $\mathcal{N}\left(E_{F}\right)$ is low throughout the series $\left(\approx 1\right.$ state $/ \mathrm{eV}$ cell $\left.{ }^{6}\right)$, the drop in $E_{F}$ is rather large ( $\sim 1 \mathrm{eV}$ as $x$ decreases from 1 to 0$)$. The nearly linear decrease in the binding energy with decreasing $x$ should cause a large increase in $T_{K}$ since

$$
k_{B} T_{K} \sim E_{F} \exp \left(-1 /|\mathcal{J}| \mathcal{N}\left(E_{F}\right)\right) \sim E_{F} \exp \left(-\left|E_{F}-E_{5 f}\right| /\left\langle V_{k f}^{2}\right\rangle \mathcal{N}\left(E_{F}\right)\right),
$$

where $V_{k f}$ is the hybridization matrix element.

From Eq. (1) it is evident that an increase in either $V_{k f}$ or $\mathcal{N}\left(E_{F}\right)$ will also cause $T_{K}$ to increase. To investigate this, we substituted other trivalent elements for $\mathrm{Y}$ to form $\mathrm{M}_{1-x} \mathrm{U}_{x} \mathrm{Pd}_{3}$, including (in order of increasing ionic radius $R$ ) $\mathrm{M}=\mathrm{Sc}$, $\mathrm{Y}, \mathrm{Pr}$, and La. For given $\mathrm{U}$ concentration $x$, a larger $R$ results in a larger lattice parameter, as shown in Fig. 2. For a Kondo system, a larger lattice constant is expected to yield a smaller hybridization strength and a corresponding smaller $T_{K}$ value. Furthermore, specific heat measurements of the host $\mathrm{MPd}_{3}$ compounds demonstrate that $\mathcal{N}\left(E_{F}\right)$ decreases with $R$, with linear coefficients $\gamma=8.20,3.48$, and $0.28 \mathrm{~mJ} / \mathrm{mol} \mathrm{K}^{2}$ for $\mathrm{M}=\mathrm{Sc}, \mathrm{Y}$, and $\mathrm{La}$, respectively. ${ }^{7}$ Note the extremely small value of $\gamma$ for $\mathrm{LaPd}_{3}$. Thermal, transport, and magnetic measurements of 

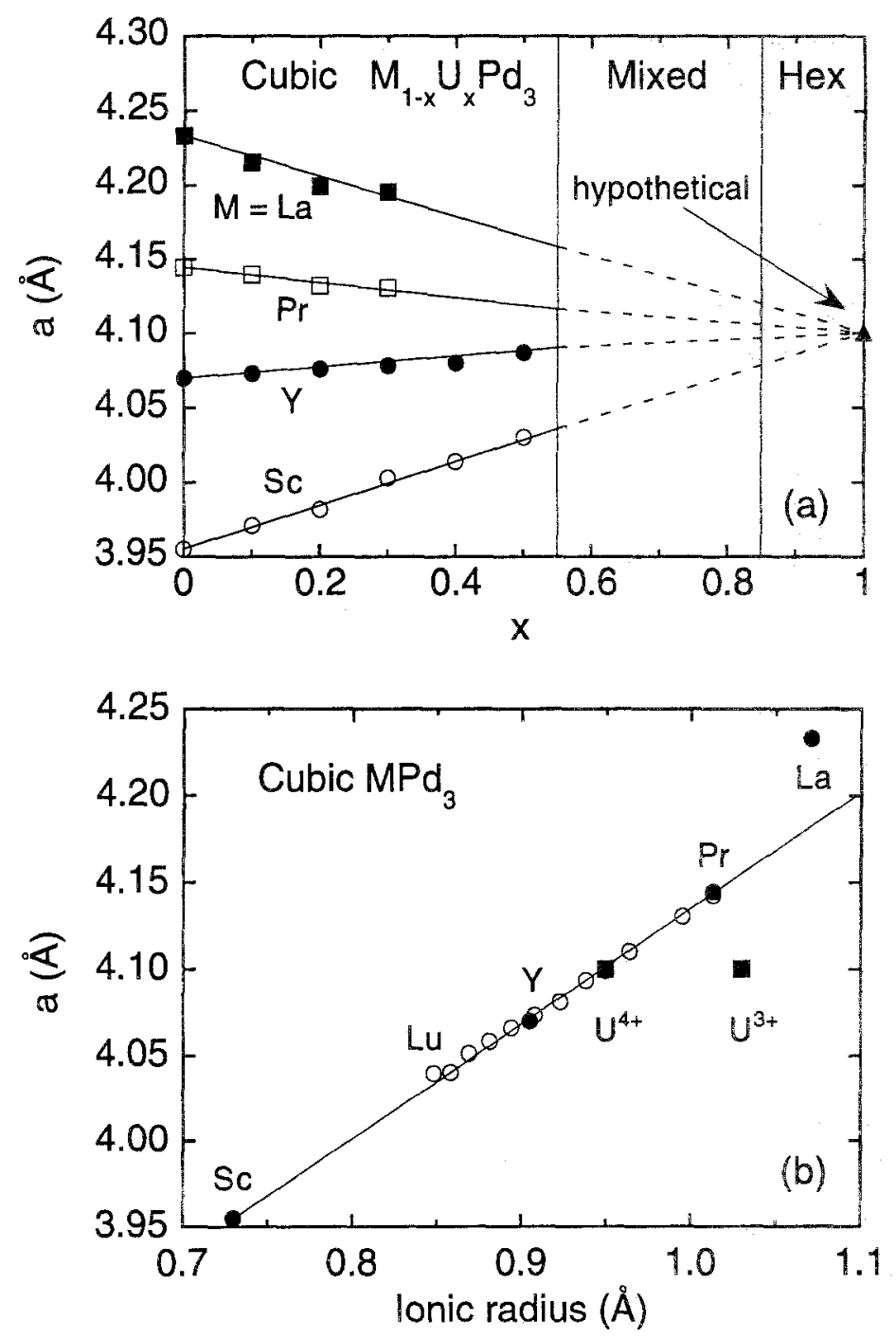

Fig. 2. (a) Cubic lattice parameters of $\mathrm{M}_{1-x} \mathrm{U}_{x} \mathrm{Pd}_{3}$ compounds as a function of $x$. Solid lines represent linear fits to the data, which extrapolate (dashed lines) to a common value of $4.100 \pm 0.005 \AA$ for hypothetical cubic UPd 3 .

(b) Lattice parameters of cubic MPd $\mathrm{d}_{3}$ compounds versus ionic radius of the trivalent ion. With the exception of $\mathrm{LaPd}_{3}$, the data fall on a straight line. The hypothetical value for cubic $\mathrm{UPd}_{3}$ obtained from (a) (filled triangle) is consistent with $U$ being tetravalent in the $\mathrm{M}_{1-x} \mathrm{U}_{x} \mathrm{Pd}_{3}$ compounds. From Ref. 8. 


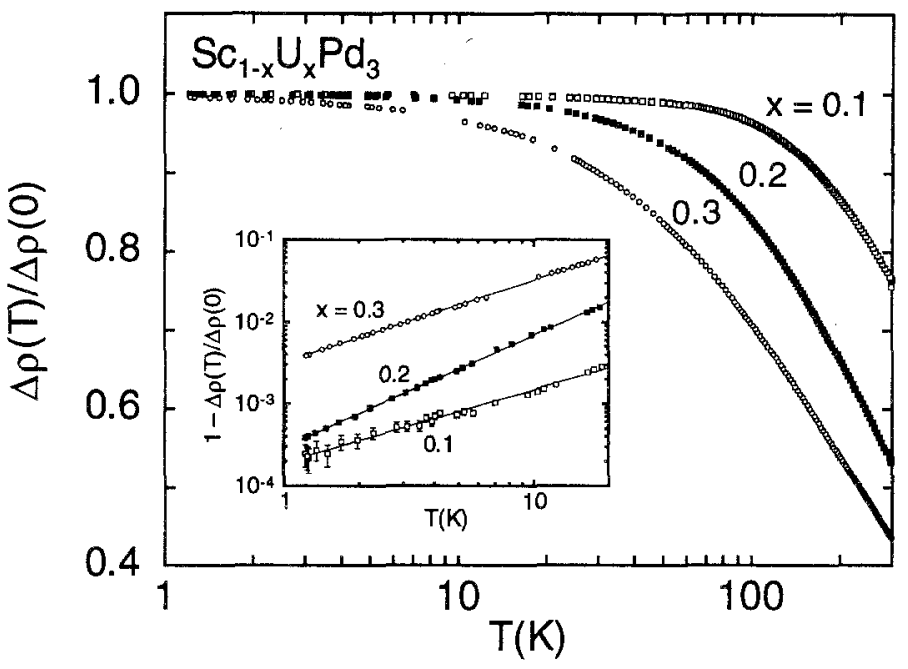

Fig. 3. Uranium contribution to the electrical resistivity $\Delta \rho(T)$ of $\mathrm{Sc}_{1-x} \mathrm{U}_{x} \mathrm{Pd}_{3}$ $(x=0.1,0.2,0.3)$, normalized to the extrapolated zero temperature value $\Delta \rho(0)$, versus $\log T$. Inset: $\log \log$ plot of $1-\Delta \rho(T) / \Delta \rho(0)=a\left(T / T_{K}\right)^{n}$ with $n=0.9 \pm 0.2$, $1.4 \pm 0.2$, and $1.0 \pm 0.2$ for $x=0.1,0.2$, and 0.3 , respectively. From Ref. 10 .

the $\mathrm{M}_{1-x} \mathrm{U}_{x} \mathrm{Pd}_{3}$ systems are indeed consistent with Fermi level tuning in which $T_{K}$ decreases with increasing $R$. Sc behaves like $\mathrm{Y}$, but with a larger $T_{K}$ for given $x$. Pr has a smaller $T_{K}$, which is barely evident as an increase in $\rho$ with decreasing $T$ for $x=1$. The La system does not display a Kondo effect at all down to at least 1 $\mathrm{K}$, for $x$ as low as 0.1 . The non Fermi liquid properties of the Kondo systems $M=$ $\mathrm{Y}$ and $\mathrm{Sc}$ are summarized below.

The observation of Fermi level tuning in cubic $\mathrm{M}_{1-x} \mathrm{U}_{x} \mathrm{Pd}_{3}$ and the absence of Fermi level tuning in hexagonal $\mathrm{M}_{1-x} \mathrm{U}_{x} \mathrm{Pd}_{3}\left(\mathrm{M}^{4+}=\mathrm{Zr}\right.$ and $\left.\mathrm{Th}\right),{ }^{9}$ imply that $\mathrm{U}$ is tetravalent in all of these $\mathrm{M}_{1-x} \mathrm{U}_{x} \mathrm{Pd}_{3}$ compounds. Further evidence for tetravalent $\mathrm{U}$ comes from considering the evolution of the cubic lattice parameters of $\mathrm{M}_{1-x} \mathrm{U}_{x} \mathrm{Pd}_{3}(\mathrm{M}=\mathrm{Sc}, \mathrm{Y}, \mathrm{La}, \mathrm{Pr} ; x \leq 0.5)$, derived from $\mathrm{X}$-ray diffraction measurements, and shown in Fig. 2. The cubic lattice parameters, plotted as a function of $x$ for each of the $\mathrm{M}_{1-x} \mathrm{U}_{x} \mathrm{Pd}_{3}$ series, extrapolate to a common value of $4.100 \pm 0.005 \AA$ for $x=1$, which represents the lattice parameter of hypothetical cubic $\mathrm{UPd}_{3}$. Comparison of this value to the lattice parameters of cubic $\mathrm{MPd}_{3}$ compounds, which when plotted as a function of $\mathrm{M}^{3+}$ ionic radius are seen to fall on a straight line, ${ }^{8}$ yield a value for the ionic radius of $U$ that is consistent with $U$ being tetravalent in $\mathrm{M}_{1-x} \mathrm{U}_{x} \mathrm{Pd}_{3}$.

\subsection{Non Fermi Liquid Properties}

The existence of a Kondo effect in the $\mathrm{M}_{1-x} \mathrm{U}_{x} \mathrm{Pd}_{3}(\mathrm{M}=\mathrm{Sc}, \mathrm{Y})$ system for $x \leq 0.3$ is evident from the electrical resistivity $\rho(T)$, which increases logarithmically 
Table 1. Approximate $T_{K}$ values (in $\mathrm{K}$ ) for $\mathrm{M}_{1-x} \mathrm{U}_{x} \mathrm{Pd}_{3}(\mathrm{M}=\mathrm{Sc}$, $\mathrm{Y}$ ), estimated as the temperature at which $\Delta \rho(T) / \Delta \rho(0)=0.8$.

\begin{tabular}{|c||c|c|c|c|c|}
\hline $\mathrm{x}$ & 0.02 & 0.05 & 0.1 & 0.2 & 0.3 \\
\hline $\mathrm{M}=\mathrm{Sc}$ & $-^{a}$ & $-^{a}$ & 260 & 120 & 60 \\
\hline $\mathrm{Y}$ & 200 & 160 & 140 & 50 & spin glass \\
\hline
\end{tabular}

Not measured.

with decreasing temperature at high temperatures $T>T_{K} \cdot{ }^{1}$ Data for $\mathrm{Sc}_{1-x} \mathrm{U}_{x} \mathrm{Pd}_{3}$ are shown in Fig. $3 .^{10}$ The $\mathrm{U}$ contribution to the resistivity $\Delta \rho(\mathrm{T})$ was estimated by subtracting $\rho(T)$ of the host $\mathrm{MPd}_{3}$ compound. Estimates of the Kondo temperature $T_{K}$ as the temperature at which the normalized resistivity $\Delta \rho(T) / \Delta \rho(0)$ equals 0.8 yield $T_{K}$ values which increase with decreasing $x$, in accordance with Fermi level tuning. Approximate values are listed in Table 1 . At low temperatures $T \ll T_{K}$, $\rho(T)$ does not saturate quadratically as

$$
\frac{\Delta \rho(T)}{\Delta \rho(0)}=1-a\left(T / T_{K}\right)^{n}
$$

with $n=2$ which is expected for a conventional Kondo effect and reflects the behavior of a Fermi liquid. Rather, we find $n \approx 1.0 \pm 0.2$ for all $\mathrm{M}=\mathrm{Y}$ samples in the temperature range $T \leq 20 \mathrm{~K}$. Power law fits for $\mathrm{M}=\mathrm{Sc}$ are shown as solid lines in the inset of Fig. 3 with slopes which correspond to $n=0.9,1.4$, and $1.0 \pm 0.2$ for $x=0.1,0.2$, and 0.3 , respectively. Taking a value for $a$ of 0.23 , values of $T_{K}$ from these power law fits vary from $\sim 40 \mathrm{~K}$ for $x=0.2$ to $\sim 500 \mathrm{~K}$ for $x=0.05$ for $\mathrm{M}=$ $\mathrm{Y}$, and from $\sim 70 \mathrm{~K}$ for $x=0.3$ to $\sim 2900 \mathrm{~K}$ for $\mathrm{M}=\mathrm{Sc}$. It is difficult to determine the power law temperature dependence of $\rho(T)$ for smaller values of $x$ because of the large values of $T_{K}$ which yield small $d \rho(T) / d T$ slopes at low temperatures.

In an attempt to approach the single impurity limit while holding $T_{K}$ approximately constant, we have made similar $\rho(T)$ measurements on the series of alloys $\mathrm{Y}_{0.8} \mathrm{Th}_{0.2-x} \mathrm{U}_{x} \mathrm{Pd}_{3}(0.01 \leq x \leq 0.2)$, shown in Fig. $4 .^{8}$ Because nonmagnetic $\mathrm{Th}$ is tetravalent, the total concentration of tetravalent ions remains fixed at 0.2 , which should yield a constant value $T_{K} \approx 40 \mathrm{~K}$ according to the Fermi level tuning scenario, everything else remaining constant. From a similar analysis of $\rho(T), T_{K}$ appears to increase slightly with decreasing $x$, as listed in Fig. 4 (a). The low temperature power law fits to Eq. (2), shown in Fig. 4 (b), again yield exponents of $n=1.0 \pm 0.1$ in the temperature range $T \leq 20 \mathrm{~K}$. The electrical resistivity thus has a NFL linear temperature dependence at the lowest measured temperatures even for samples with $\mathrm{U}$ concentrations as low as $1 \%$. The $\mathrm{U}$ concentration independence of this feature suggests that it is not due to interimpurity interactions.

Recently, an $\mathrm{Y}_{0.8} \mathrm{U}_{0.2} \mathrm{Pd}_{3}$ polycrystalline specimen was investigated optically over a broad frequency range from 15 to $10^{5} \mathrm{~cm}^{-1}$ by Degiorgi et al. ${ }^{11}$ Evaluation of the frequency dependence of the transport relaxation rate $\tau^{-1}$ at several temperatures reveals that $\tau^{-1}$ increases linearly with decreasing frequency and temperature. This was interpreted as another manifestation of NFL behavior in the $\mathrm{Y}_{1-x} \mathrm{U}_{x} \mathrm{Pd}_{3}$ system.

Shown in Fig. 5 is the uranium contribution to the specific heat $\Delta C$, plotted as 

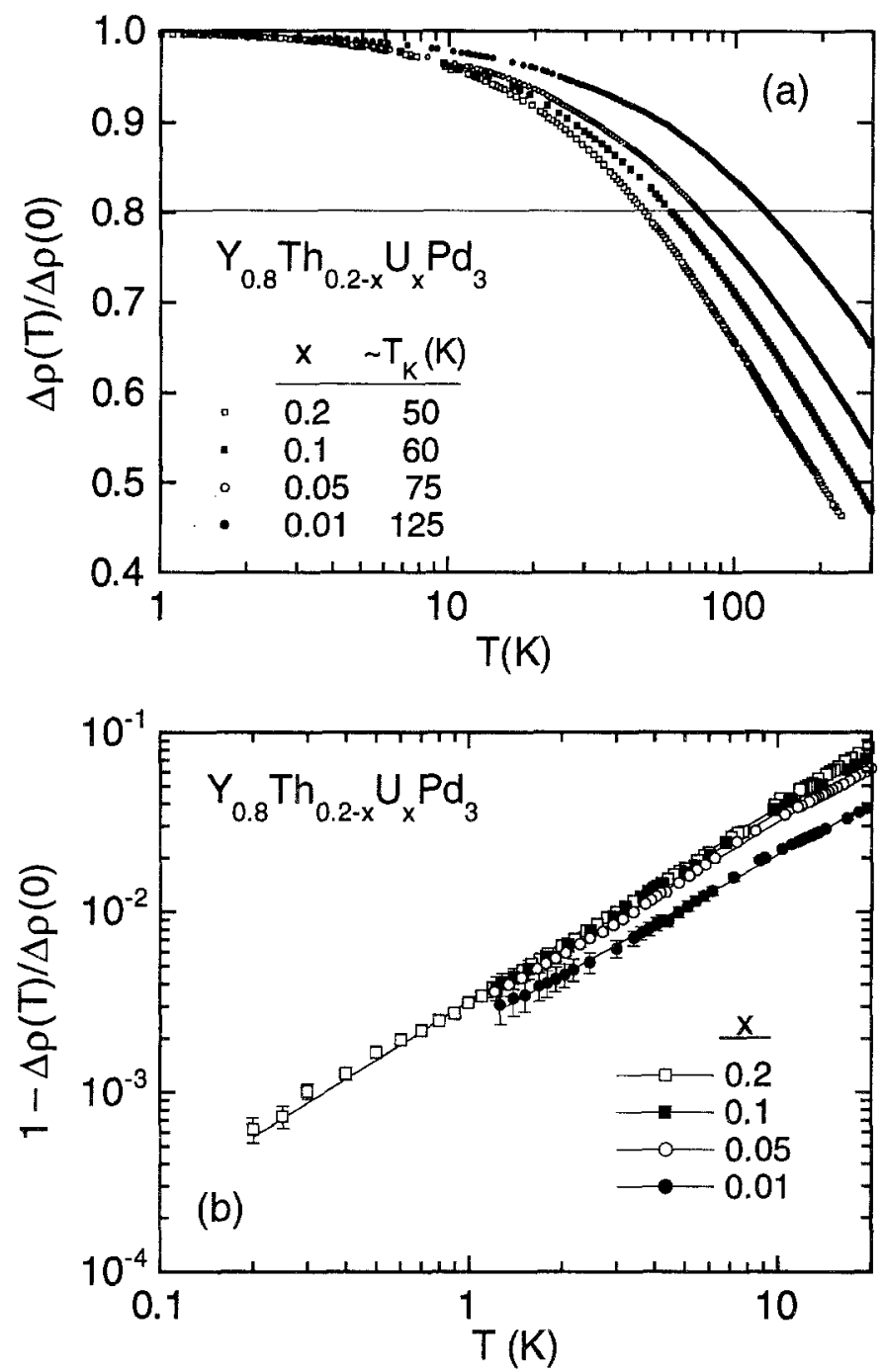

Fig. 4. (a) Normalized electrical resistivity $\Delta \rho(T) / \Delta \rho(0)$ versus $\log T$ for the $\mathrm{Y}_{0.8} \mathrm{Th}_{0.2-x} \mathrm{U}_{x} \mathrm{Pd}_{3}(0 \leq x \leq 0.2)$ system revealing Kondo behavior with a Kondo temperature $T_{K}$ which increases only slightly with decreasing $x$.

(b) Low temperature resistivity data $(T \leq 20 \mathrm{~K})$ plotted as $\log (1-\Delta \rho(T) / \Delta \rho(0))$ versus $\log T$. Solid lines represent fits to a power law $\Delta \rho(T) / \Delta \rho(0)=1-a\left(T / T_{K}\right)^{n}$, with $n=1.0 \pm 0.1$ for all samples, exhibiting NFL behavior. This value is in between that expected for single channel $(n=2)$ and two channel $(n=1 / 2) S=1 / 2$ Kondo models. From Ref. 8. 


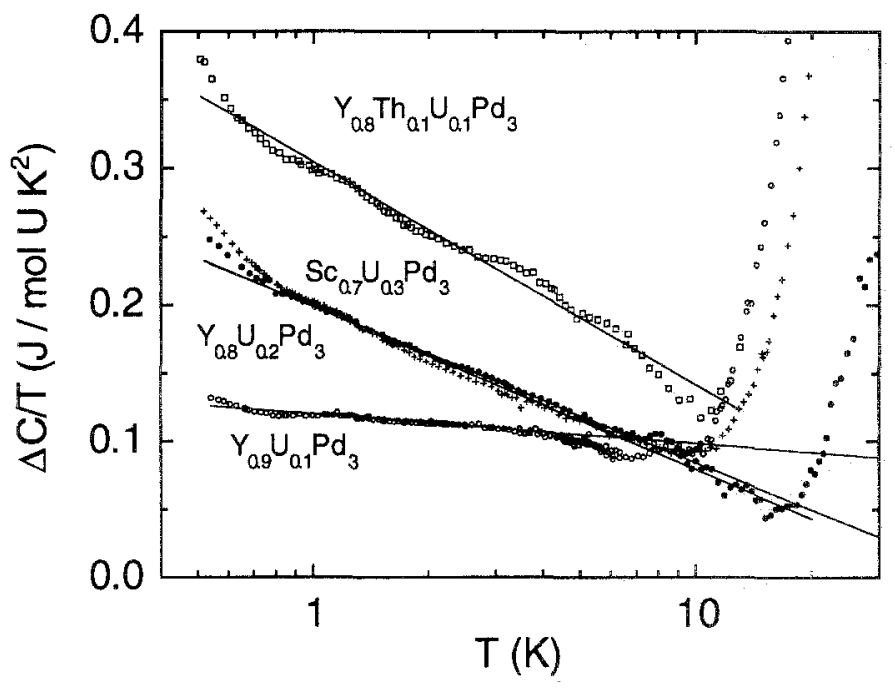

Fig. 5. Low temperature specific heat $\Delta C / T$ versus $\log T$ for various Kondo alloys which exhibit a $T=0$ logarithmic divergence, a non Fermi liquid behavior. Solid lines are fits of the data to the two channel, $S=1 / 2$ Kondo model plus a constant $B^{\prime}: \Delta C / T=-\left(0.251 / T_{K}\right) R \ln \left(T / 0.41 T_{K}\right)+B^{\prime}$. The associated entropy suggests a finite zero temperature entropy of $S(0) \approx(R / 2) \ln (2)$. From Ref. 8 .

$\Delta C / T$ versus $\log T$, for several samples which display a characteristic logarithmic divergence over the temperature range $0.7 \mathrm{~K} \lesssim T \lesssim 10 \mathrm{~K}$, including $\mathrm{Y}_{0.8} \mathrm{U}_{0.2} \mathrm{Pd}_{3}$, $\mathrm{Y}_{0.9} \mathrm{U}_{0.1} \mathrm{Pd}_{3}, \mathrm{Y}_{0.8} \mathrm{Th}_{0.1} \mathrm{U}_{0.1} \mathrm{Pd}_{3}$, and $\mathrm{Sc}_{0.7} \mathrm{U}_{0.3} \mathrm{Pd}_{3} .^{8}$ Such NFL behavior is in striking contrast to a conventional Kondo effect where $\Delta C / T$ approaches a constant value which varies as $1 / T_{K}$ at low temperatures $T \ll T_{K}$. At higher temperatures, the data increase sharply due to difficulties in subtracting the phonon contribution and/or a Schottky anomaly arising from an excited state CEF level above the ground state. The solid lines represent least squares linear fits of the data to the equation $\Delta C / T=A \ln T+B$. (Here, ln represents the natural logarithm). Irrespective of the microscopic mechanism responsible for such NFL behavior, it is useful to investigate how the data scale with the characteristic energy of the system, the Kondo temperature $T_{K}$, according to

$$
\Delta C / T=\left(-A^{\prime} R / T_{K}\right) \ln \left(T / b T_{K}\right)+B^{\prime}
$$

where $A^{\prime}, b$, and $B^{\prime}$ are constants and $R$ is the gas constant. If we assume this form of scaling with $T_{K}$, larger logarithmic slopes (coefficient $A$ ) correspond to smaller values of $T_{K}$. Using values $b=0.41$ and $A^{\prime}=0.251$ (the value for a two channel Kondo effect), one can extract a value for $T_{K}$ from the slope $A$ of these lines, and $B^{\prime}$ from the offset $B$. Values for $B^{\prime}$ are comparable to measured values of the linear coefficient of the specific heat $\gamma$ for the host metals: $\gamma \approx 3.5,6.9$, and $8.2 \mathrm{~mJ} / \mathrm{mol} \mathrm{K}{ }^{2}$ for $\mathrm{YPd}_{3},{ }^{7} \mathrm{Y}_{0.8} \mathrm{Th}_{0.2} \mathrm{Pd}_{3}$, and $\mathrm{ScPd}_{3},{ }^{7}$ respectively, which have not been subtracted from the data. A hybridization broadened Schottky anomaly 


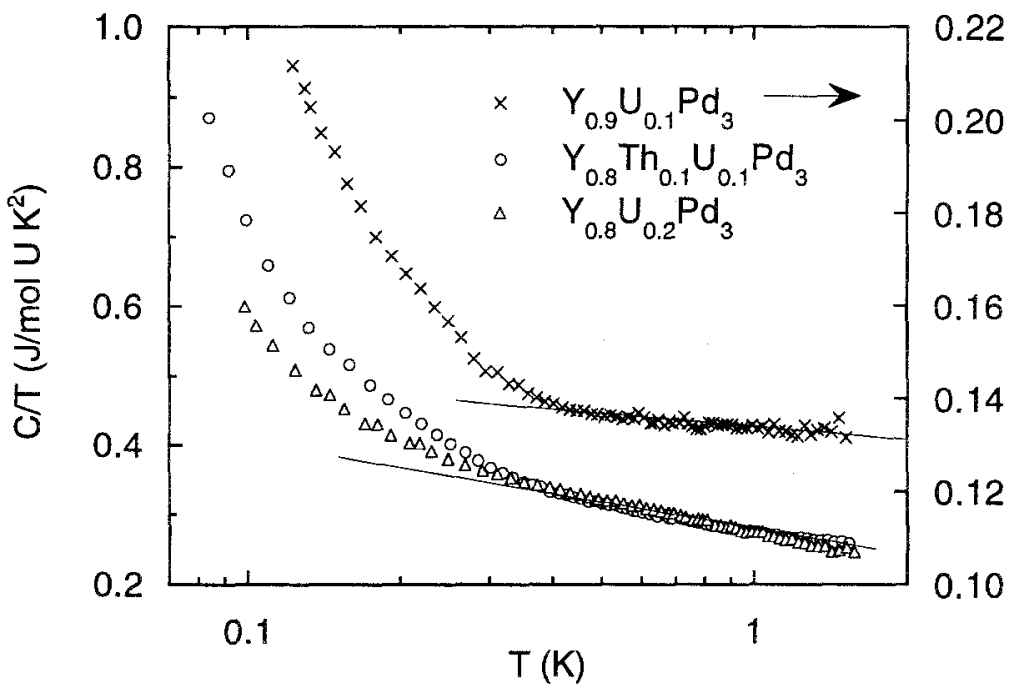

Fig. 6. Specific heat $C / T$ versus $\log T$ to temperatures as low as $80 \mathrm{mK}$ for three of the Kondo alloys from Fig. 5, measured in a ${ }^{3} \mathrm{He}-{ }^{4} \mathrm{He}$ dilution refrigerator. Solid lines are fits of the data to the two channel, $S=1 / 2$ Kondo model plus a constant $B^{\prime}$.

at higher temperatures can also contribute to $B^{\prime}$. Especially noteworthy is the behavior of the more dilute compound $\mathrm{Y}_{0.8} \mathrm{Th}_{0.1} \mathrm{U}_{0.1} \mathrm{Pd}_{3}$ which is nearly identical to that of $\mathrm{Y}_{0.8} \mathrm{U}_{0.2} \mathrm{Pd}_{3}$, which suggests that the observed NFL behavior is not due to interimpurity interactions which decrease with decreasing $U$ concentration, but is dominated by single impurity effects. Parameter values for $\mathrm{Sc}_{0.7} \mathrm{U}_{0.3} \mathrm{Pd}_{3}$ are also comparable to those for $\mathrm{Y}_{0.8} \mathrm{U}_{0.2} \mathrm{Pd}_{3}$. Fits of the $\Delta C / T$ data for $\mathrm{Y}_{0.9} \mathrm{U}_{0.1} \mathrm{Pd}_{3}$ yield a larger $T_{K}$ value than for $\mathrm{Y}_{0.8} \mathrm{U}_{0.2} \mathrm{Pd}_{3}$, in agreement with the resistivity data and Fermi level tuning mechanism.

A remarkable aspect of the specific heat data is that the associated entropy $S(T)$ for all of these samples saturates to a value close to $(R / 2) \ln (2)$ before continuing to increase due to the higher temperature upturns in $\Delta C / T$ seen in Fig. $5{ }^{1}$ This suggests a finite zero temperature entropy of the same value, in order that the full degeneracy of the ground doublet be recovered at high temperatures. Such an unusual $T=0$ entropy is predicted for the two channel, $S=1 / 2 \mathrm{Kondo}_{\text {model }} .^{12}$ However, this entropy is expected to be removed at low but finite temperatures due to any weak interactions which might lift the ground state degeneracy.

The results of recent heat capacity measurements on several samples in the $\mathrm{Y}_{1-x}\left(\mathrm{Th}_{y} \mathrm{U}_{1-y}\right)_{x} \mathrm{Pd}_{3}$ series taken in a ${ }^{3} \mathrm{He}^{-}{ }^{4} \mathrm{He}$ dilution refrigerator to temperatures as low as $80 \mathrm{mK}$ are shown in Fig. 6. The logarithmic temperature dependence of $C / T$ persists down to about $0.5 \mathrm{~K}$. Straight lines through the $C / T$ data above $0.5 \mathrm{~K}$ over the limited temperature range shown correspond to $T_{K}=40 \mathrm{~K}$, 
$B^{\prime}=0.132$, for $\mathrm{Y}_{0.8} \mathrm{U}_{0.2} \mathrm{Pd}_{3}$ and $\mathrm{Y}_{0.8} \mathrm{Th}_{0.1} \mathrm{U}_{0.1} \mathrm{Pd}_{3}$ and $T_{K}=680 \mathrm{~K}, B^{\prime}=0.117$, for $\mathrm{Y}_{0.9} \mathrm{U}_{0.1} \mathrm{Pd}_{3}$. The $C / T$ data for $\mathrm{Y}_{0.8} \mathrm{U}_{0.2} \mathrm{Pd}_{3}$ in Fig. 6 are somewhat higher than the corresponding data in Fig. 5 ; the origin of this discrepancy is presently under investigation. Below $\sim 0.5 \mathrm{~K}, C / T$ rises dramatically without any sign of saturation down to the lowest temperature studied. This behavior is similar to that previously found by $\mathrm{Ott}$ et al. for $\mathrm{Y}_{0.8} \mathrm{U}_{0.2} \mathrm{Pd}_{3}$ who reported that the upturn in $C / T$ persisted to at least $0.05 \mathrm{~K} .^{13}$ The deviation away from logarithmic dependence is well described by $\Delta C / T=A T^{-2}$, which is the temperature dependence of a Schottky anomaly in the high temperature limit. The magnitude of $A$ from the measurements of Ott et al. ${ }^{13}$ is too large for a nuclear Schottky anomaly, but for a two level electronic Schottky anomaly corresponds to a level splitting of $2.5 \mathrm{mK}$. It has been suggested previously ${ }^{8}$ that this rise may account for the missing entropy of $\sim(R / 2) \ln (2)$. Our results are consistent with this proposition.

The magnetic susceptibility of $\mathrm{M}_{1-x} \mathrm{U}_{x} \mathrm{Pd}_{3}(\mathrm{M}=\mathrm{Sc}, \mathrm{Y}, \mathrm{La})$, as well as that of $\mathrm{Y}_{1-x}\left(\mathrm{Th}_{y} \mathrm{U}_{1-y}\right)_{x} \mathrm{Pd}_{3}$, exhibits a Curie-Weiss temperature dependence $N \mu_{e f f}^{2} / 3 k_{B}\left(T-\theta_{p}\right)$, where $N$ is the number of $\mathrm{U}$ ions, $\mu_{e f f}$ is the $\mathrm{U}$ effective moment, and $\theta_{p}$ is the Curie-Weiss temperature, at high temperatures $T>150 \mathrm{~K}$, after subtraction of a temperature independent background term $\chi_{0} .{ }^{1}$ The effective moment is close to the free ion value for either tetravalent or trivalent $U$. The Curie-Weiss temperature $\theta_{p}$ is large and negative, indicative of antiferromagetic exchange interactions, with a magnitude that correlates with the Kondo temperature such that $\left|\theta_{p}\right| \approx 4 T_{K}$, typical of Kondo systems. The $T_{K}$ inferred from $\theta_{p}$ is associated with the antiferromagnetic exchange interaction between the conduction electrons and the excited magnetic states which are thermally populated at higher temperatures.

At low temperatures, $\chi(T)$ of $\mathrm{M}_{1-x} \mathrm{U}_{x} \mathrm{Pd}_{3}(\mathrm{M}=\mathrm{Y}, \mathrm{Sc})$ increases more rapidly with decreasing temperature than the Curie-Weiss temperature dependence, and continues to increase with positive curvature down to the lowest measured temperature for $\mathrm{x} \leq 0.2$ and 0.3 for $\mathrm{M}=\mathrm{Y}$ and $\mathrm{Sc}$, respectively. This is in contrast to the Fermi liquid behavior of the single channel, $S=1 / 2$ Kondo effect in which $\chi(T)$ becomes temperature independent for $T \ll T_{K}$ due to screening of the impurity moments by the conduction electrons. The precise temperature dependence of $\chi(T)$ at the lowest temperatures is masked by the possible presence of magnetic impurities other than $U$. We have estimated and subtracted the impurity contribution of $\mathrm{Y}_{0.8} \mathrm{U}_{0.2} \mathrm{Pd}_{3}$ through analysis of isothermal $M(H)$ curves as described elsewhere, ${ }^{2}$ yielding the intrinsic susceptibility at various temperatures from $0.6 \mathrm{~K}$ to $40 \mathrm{~K}$ shown in Fig. 7 . The $\chi(T)$ data are well described by the equation

$$
\chi(T)=\chi(0)\left(1-c\left(T / T_{K}\right)^{1 / 2}\right)
$$

rèpresented by the solid line in Fig. 7 . The data appear to exhibit a $T^{1 / 2}$ temperature dependence over a wide temperature range, down to the lowest temperatures, extrapolating to a value of $5.9 \times 10^{-3} \mathrm{emu} / \mathrm{mol} \mathrm{U}$ at $T=0$. If we assume $T_{K}=$ $42 \mathrm{~K}$, then from the fit we obtain $c=0.36$.

$A$ number of other measurements have been performed on the $\mathrm{Y}_{1-x} \mathrm{U}_{x} \mathrm{Pd}_{3}$ system in order to further characterize and explore the NFL behavior in this system. Thermoelectric power $S(T)$ measurements from $2-200 \mathrm{~K}$ have been made in 


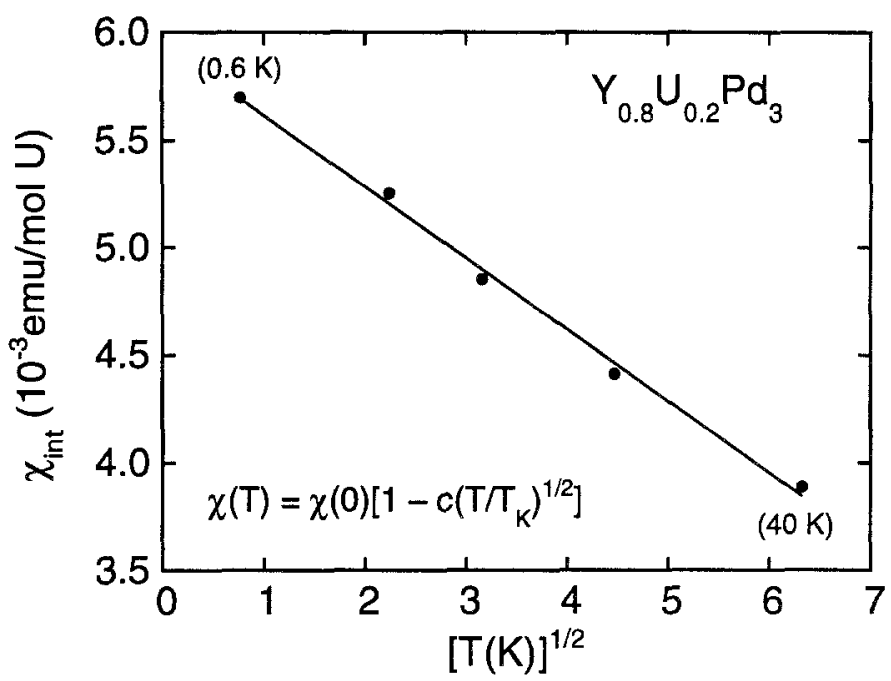

Fig. 7. Intrinsic magnetic susceptibility $\chi_{i n t}$ versus square root of the temperature for $\mathrm{Y}_{0.8} \mathrm{U}_{0.2} \mathrm{Pd}_{3}$. The solid line represents a fit of the data to the equation $\chi(T)=$ $\chi(0)\left(1-c\left(T / T_{K}\right)^{1 / 2}\right)$, where $\chi(0)=5.9 \times 10^{-3} \mathrm{emu} / \mathrm{mol} \mathrm{U}$ and $c=0.36$ for $T_{K}=$ 42 K. From Ref. 2.

$\mathrm{Y}_{1-x} \mathrm{U}_{x} \mathrm{Pd}_{3}$ in the range $0 \leq x \leq 0.4 .^{14}$ The behavior of $S(T)$ for $x=0.05,0.1$ is reminiscent of typical Kondo materials in the sense that there is a "giant" positive contribution linear in $T$ at low temperatures and independent of $x$. However, this result suggests that $T_{K}$ is independent of $x$, in contrast to the $\rho(T), C(T)$, and $\chi(T)$ measurements discussed herein. At high temperatures, $S(T)$ changes from positive to negative as $x$ is varied from 0.1 to 0.3 , suggestive of a cross-over or transition in electronic structure near $x=0.2$. The spin-glass transition of the $x=0.3,0.4$ alloys is signaled by a zero-crossing of $S$, similar to that observed for conventional spin glasses.

\subsection{Fermi Level Tuning in the $\left(\mathbf{Y}_{1-y} \mathrm{Th}_{y}\right)_{1-x} \mathbf{U}_{x} \mathbf{P d}_{3}$ System}

Besides Fermi level tuning, the substitution of $\mathrm{U}^{4+}$ for $\mathrm{Y}^{3+}$ in the $\mathrm{Y}_{1-x} \mathrm{U}_{x} \mathrm{Pd}_{3}$ system has the additional effect of increasing the $U$ intersite interaction strength as $x$ is increased which leads to spin-glass freezing below a characteristic temperature $T_{S G}$ that increases with $x$. In order to study the effect of the increase of $\left|E_{F}-E_{5 f}\right|$ on $T_{K}$, with $\mathrm{U}$ intersite interactions held constant to first approximation, we have performed measurements of $\rho(T), \chi(T)$, and $C(T)$ as functions of $y$ for a fixed value $\mathrm{x}=0.1$ in the $\left(\mathrm{Y}_{1-y} \mathrm{Th}_{y}\right)_{1-x} \mathrm{U}_{x} \mathrm{Pd}_{3}$ system. ${ }^{15}$ The data clearly reveal the scaling of the physical properties with $T_{K}$ and the phenomenon of Fermi level tuning in this system.

Shown in Fig. 8(a) are plots of $\rho$ versus $T$ between $1.2 \mathrm{~K}$ and $300 \mathrm{~K}$ for $\mathrm{Y}_{0,9-y} \mathrm{Th}_{y} \mathrm{U}_{0.1} \mathrm{Pd}_{3}$ samples with $y=0,0.1,0.2,0.3$, and 0.4 . The $\rho(T)$ data for 0 $\leq \mathrm{y} \leq 0.2$ reveal that $\rho$ increases with decreasing $T$, indicative of a Kondo effect. 

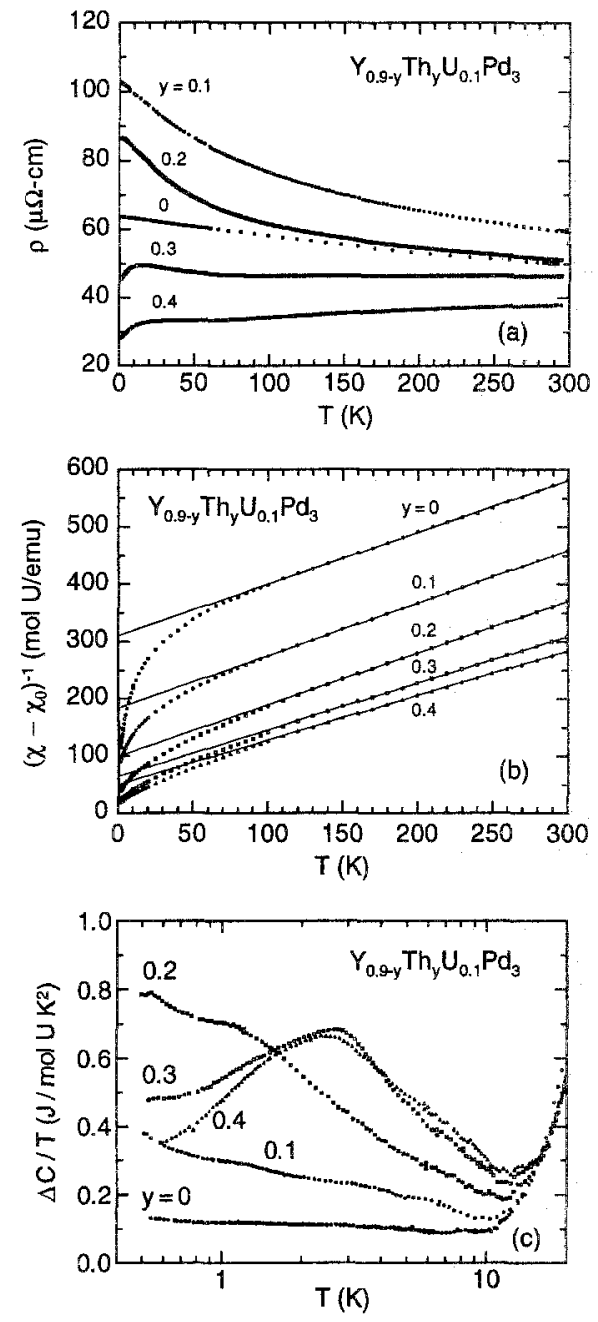

Fig. 8. Temperature $T$ dependence of the electrical resistivity $\rho$, magnetic susceptibility $\chi$, and electronic specific heat $\Delta C$ of the $\mathrm{Y}_{0.9-y} \mathrm{Th}_{y} \mathrm{U}_{0.1} \mathrm{Pd}_{3}(y=0,0.1,0.2$, $0.3,0.4)$ system: (a) $\rho$ versus $T$; (b) $\left(\chi-\chi_{0}\right)^{-1}$ versus $T$, where $\chi_{0}$ is defined in the text; (c) $\Delta C / T$ versus $\ln T$. From Ref. 15 . 
The increase of the temperature dependence of $\rho$ with $y$ for $0 \leq y \leq 0.2$ suggests that $T_{K}$ decreases with $y$, consistent with Fermi level tuning.

Displayed in Fig. $8(\mathrm{~b})$ are plots of $\left(\chi-\chi_{0}\right)^{-1}$ versus $T$ between $1.8 \mathrm{~K}$ and $300 \mathrm{~K}$, where $\chi_{0}$ was determined by fitting the $\chi(T)$ data to the sum of a constant $\chi_{0}$ and a Curie-Weiss law. Below $\sim 100 \mathrm{~K}$, the $\left(\chi-\chi_{0}\right)^{-1}$ versus $T$ data fall below the linear fits to the higher $T$ data and approach a finite value as $T \rightarrow 0$, indicative of a nonmagnetic ground state, which is presumably the $\mathrm{U} \Gamma_{3}$ nonmagnetic doublet. The magnitude of the negative Curie-Weiss temperature $\left|\theta_{p}\right|$ decreases exponentially and saturates to a value of $57 \mathrm{~K}$ for large $y$. A comparable $x$-independent value of $\theta_{p}$ was observed for the related system $\mathrm{La}_{1-x} \mathrm{U}_{x} \mathrm{Pd}_{3}$ which does not show a Kondo effect. This decrease is consistent with the decrease of $T_{K}$ with $y$ inferred from the $\rho(T)$ data of Fig. 8(a) since for Kondo systems, $\left|\theta_{p}\right| \approx 3-4 T_{K}$.

Presented in Fig. 8(c) are plots of $\Delta C / T$ versus $\ln T$ for $\mathrm{Y}_{0.9-y} \mathrm{Th}_{y} \mathrm{U}_{0.1} \mathrm{Pd}_{3}$ between $\sim 0.5 \mathrm{~K}$ and $20 \mathrm{~K}$. The increase of the magnitude of the slope $|d(\Delta C / T) / d \ln T|$ with $y$ is consistent with a decrease of $T_{K}$ with $y$. The features in the $\Delta C / T$ versus $\ln T$ data at $\sim 1 \mathrm{~K}$ (a shoulder) for the sample with $y=0.2$ and at $\sim 2.4 \mathrm{~K}$ (a maximum) for the samples with $x=0.3$ and 0.4 may be due to the splitting of the $\Gamma_{3}$ nonmagnetic doublet ground state, which is analogous to Zeeman splitting of the magnetic doublet for a magnetic two-channel Kondo effect. It is interesting that the evolution of the shape of the $\Delta C(T)$ curves with $y$ resembles that of the calculated $\Delta C(T)$ curves for a two channel magnetic Kondo effect in the presence of an applied magnetic field. ${ }^{16}$ Such a splitting of the $U \Gamma_{3}$ doublet could arise from a local charge asymmetry or distortion of the lattice from cubic symmetry about a $U$ site due to the substitution of $T h$. An analysis of the slope $|d(\Delta C / T) / d \ln T|$ within the context of the two channel Kondo model yields a decrease of $T_{K}$ with $y$, similar to that deduced from the $\chi(T)$ data.

\subsection{Crystalline Electric Field Energy Level Scheme for $\mathrm{U}^{4+}$}

In order to understand the microscopic origin of the observed NFL behavior in the thermal, transport, and magnetic properties, it is important to determine the CEF energy level scheme of the $U 5$ f electrons. Tetravalent $U$ ions have two localized 5 f electrons $\left(5 f^{2}\right)$, which have a Hund's rules magnetic ground state multiplet configuration ${ }^{3} \mathrm{H}_{4}(S=1, L=5, J=4)$. In a cubic CEF, the 9-fold degeneracy is lifted, leaving a $\Gamma_{1}$ singlet, $\Gamma_{4}$ and $\Gamma_{5}$ triplets, and a $\Gamma_{3}$ nonmagnetic (non Kramers) doublet. ${ }^{17}$ According to Lea, Leask, and Wolf, it is possible for either the $\Gamma_{1}, \Gamma_{3}$, or $\Gamma_{5}$ state to lie lowest in energy. These states, in turn, can hybridize with conduction electron states leading to Kondo, heavy fermion, or intermediate valence behavior. For actinide compounds, the CEF splitting and hybridization energy scales are often comparable and can range from $1 \mathrm{~K}$ to $10^{3} \mathrm{~K}$.

Previous inelastic neutron scattering measurements on $\mathrm{Y}_{0.8} \overline{\mathrm{U}}_{0.2} \mathrm{Pd}_{3}$ at $10 \mathrm{~K}$ revealed two magnetic peaks centered at energies $\mathrm{E} \approx 5 \mathrm{meV}$ and $16 \mathrm{meV}$, attributed to hybridization broadened CEF levels, with integrated intensities consistent with $\Gamma_{5}$ and $\Gamma_{4}$ first and second excited state triplets, respectively, lying above a $\Gamma_{3}$ doublet ground state. ${ }^{18}$ For a magnetic Kondo effect, one expects to observe quasielastic scattering with a half width of the order of $k_{B} T_{K}$, which for $\mathrm{Y}_{0.8} \mathrm{U}_{0.2} \mathrm{Pd}_{3}$ is at least $4 \mathrm{meV}$. The absence of a pronounced low energy quasielastic response provided 
further evidence that the nonmagnetic $\Gamma_{3}$ doublet lies lowest in energy.

The possibility of a low energy quasielastic response was investigated further by performing inelastic neutron scattering at low energies. Low temperature $(\sim 1.5 \mathrm{~K})$ data with $\sim 0.5 \mathrm{meV}$ resolution showed no indication of substantial scattering below the first $\mathrm{CEF}$ peak at $\sim 7 \mathrm{meV}$, while higher resolution $(0.1 \mathrm{meV})$ measurements up to $0.6 \mathrm{meV}$ showed no scattering above $\sim 0.2 \mathrm{meV}$. In an attempt to identify the magnetic scattering even at zero energy transfer, polarized measurements of the spin flip scattering by subtracting the spectra taken using a vertical field from that using a horizontal field are in progress.

The $\Gamma_{3}$ state consists of two degenerate singlet states which can be expressed as linear combinations of the $J_{z}$ eigenstates $\left|J_{z}\right\rangle$ as $0.5401(|4\rangle+|-4\rangle)-0.6455|0\rangle$ and $(1 / \sqrt{2})(|2\rangle+|-2\rangle)$. It is apparent that the magnetic moment, which is proportional to the expectation value of $J_{z},\left\langle\Gamma_{3}\left|J_{z}\right| \Gamma_{3}\right\rangle$, vanishes. However, the expectation value of the $\mathrm{z}$-component of the electric quadrupole moment, which is proportional to $\left\langle\Gamma_{3}\left|3 J_{z}^{2}-J(J+1)\right| \Gamma_{3}\right\rangle$, is finite. Thus, an electric field gradient, which can be obtained through the application of uniaxial stress or some other interaction which drives the system away from CEF cubic symmetry, will induce a net electric quadrupole moment on the impurity in which the charge distribution of the $5 \mathrm{f} \mathrm{elec-}$ trons in the $\Gamma_{3}$ state no longer has spherical symmetry. It is this charge asymmetry that the conduction electrons overcompensate through the two channel quadrupolar Kondo effect (QKE). While it is not clear that a QKE is responsible for the NFL low temperature behavior in the $\mathrm{Y}_{1-x} \mathrm{U}_{x} \mathrm{Pd}_{3}$ system, the ground state does appear to be a $\Gamma_{3}$ nonmagnetic doublet with an electric quadrupole moment so that the conditions for a QKE seem to be satisfied. However, recent elastic constant measurements on a polycrystalline specimen of $\mathrm{Y}_{0.8} \mathrm{U}_{0.2} \mathrm{Pd}_{3}$ by Amara et al. ${ }^{19}$ revealed no strong variation with $T$ as expected for a quadrupolar interaction.

\section{THE $\mathrm{U}_{1-x} \mathbf{T h}_{x} \mathbf{P d}_{2} \mathrm{Al}_{3}$ SYSTEM}

In 1991, Geibel et al. discovered two new U-based heavy fermion superconductors, $\mathrm{UNi}_{2} \mathrm{Al}_{3}{ }^{20}$ and $\mathrm{UPd}_{2} \mathrm{Al}_{3},{ }^{21}$ which crystallize in the hexagonal $\mathrm{PrNi}_{2} \mathrm{Al}_{3}$ structure. These two compounds exhibit the coexistence of superconductivity and antiferromagnetism $(\mathrm{AFM})$ with $T_{c}<T_{N}$; for $\mathrm{UNi}_{2} \mathrm{Al}_{3}, T_{c} \approx 1 \mathrm{~K}$ and $T_{N}=4.6 \mathrm{~K}$, while for $\mathrm{UPd}_{2} \mathrm{Al}_{3}, T_{c} \approx 2 \mathrm{~K}$ and $T_{N}=14.6 \mathrm{~K}$.

The compound $\mathrm{UPd}_{2} \mathrm{Al}_{3}$ has the highest $T_{c}$ of the heavy fermion superconductors and a large ordered magnetic moment of $0.85 \mu_{B},{ }^{22}$ in contrast to the small ordered moments $\left(\sim 0.02 \mu_{B}\right)$ observed for $\mathrm{URu}_{2} \mathrm{Si}_{2}{ }^{23}$ and $\mathrm{UPt}_{3},{ }^{24}$ two other heavy fermion compounds in which superconductivity and AFM coexist with $T_{c}<T_{N}$. The antiferromagnetic structure of $\mathrm{UPd}_{2} \mathrm{Al}_{3}$ consists of alternating ferromagnetic sheets, with the moments lying in the hexagonal basal plane. ${ }^{22}$ The temperature dependence of the upper critical field is consistent with singlet superconductivity and the antiferromagnetic transition appears to involve the opening of a $30 \mathrm{meV}$ gap over part of the Fermi surface, ${ }^{25}$ similar to that observed in $\mathrm{URu}_{2} \mathrm{Si}_{2} .{ }^{26}$

In an effort to find new examples of NFL behavior, we embarked on an investigation of the $\mathrm{U}_{1-x} \mathrm{Th}_{x} \mathrm{Pd}_{2} \mathrm{Al}_{3}$ system about a year ago. Our initial studies revealed NFL behavior in the low temperature physical properties of this system ${ }^{2,27}$ which we have characterized in more detail during the past year. Based upon measurements 


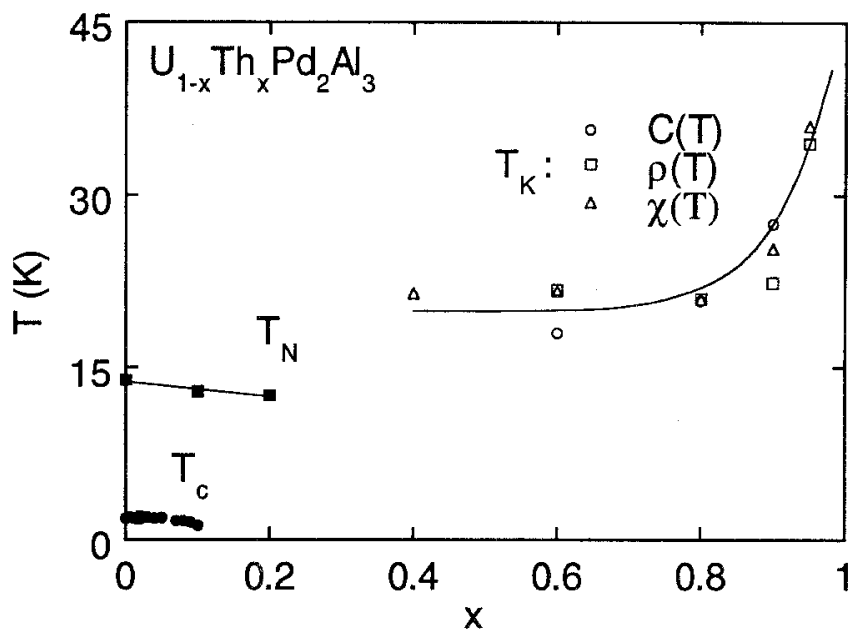

Fig. 9. Low temperature phase diagram of the $\mathrm{U}_{1-x} \mathrm{Th}_{x} \mathrm{Pd}_{2} \mathrm{Al}_{3}$ system. As the Th concentration $x$ is increased, the Neél temperature $\mathrm{T}_{N}$ and the superconducting critical temperature $T_{c}$ decrease slightly, but the features associated with AFM and superconductivity are rapidly suppressed and eventually become undetectable. The line in the right hand side of the figure represents the estimated value of the Kondo temperature $\mathrm{T}_{K}$.

of $\rho(T), C(T)$, and $\chi(T)$, which will be briefly reviewed below, the low temperature phase diagram of the $\mathrm{U}_{1-x} \mathrm{Th}_{x} \mathrm{Pd}_{2} \mathrm{Al}_{3}$ system shown in Fig. 9 has been established. However, this phase diagram is incomplete near $x=1$ since we have discovered during the course of this investigation that the compound $\mathrm{ThPd}_{2} \mathrm{Al}_{3}$ is superconducting with a $T_{c}=0.2 \mathrm{~K}$. This is an extraordinary result, since it is the first case where a $\mathrm{Ce}$ or $\mathrm{U}$ heavy fermion superconductor has an isostructural counterpart based on a rare earth or actinide element with an empty or filled f-electron shell (i. e., Sc, Y, La, Lu, Th) which is also superconducting. We are presently measuring the $T_{c}(x)$ curve for the $\mathrm{U}_{1-x} \mathrm{Th}_{x} \mathrm{Pd}_{2} \mathrm{Al}_{3}$ system near $x=1$.

Electrical resisitivity $\rho(T)$ data for the $\mathrm{U}_{1-x} \mathrm{Th}_{x} \mathrm{Pd}_{2} \mathrm{Al}_{3}$ system for various values of $x$ between 0 and 1 are shown in Fig. 10. With increasing $x$, the Neél temperature $T_{N}$ remains nearly constant up to $x=0.4$, while the drop in $\rho$ below $T_{N}$ is rapidly suppressed. For values of $x \geq 0.6$, no magnetic ordering is evident and $\rho(T)$ exhibits single ion Kondo like behavior. However, $\rho(T)$ is nearly linear over an appreciable temperature range between about $\sim 4 \mathrm{~K}$ and $\sim 30 \mathrm{~K}$, indicative of $\mathrm{NFL}$ behavior and similar to that observed for the $\mathrm{M}_{1-x} \mathrm{U}_{x} \mathrm{Pd}_{3}(\mathrm{M}=\mathrm{Sc}, \mathrm{Y})$ systems discussed in the preceding section. The resistivity $\rho(T)$ levels of below $\sim 4 \mathrm{~K}$, suggesting that the degeneracy of the conduction electron channels or the localized electron spin or charge degrees of freedom has been removed by some type of residual interaction, producing an evolution towards single channel FL behavior.

Plots of $\log (1-\Delta \rho(T) / \Delta \rho(0))$ versus $\log T$ between $0.1 \mathrm{~K}$ and $20 \mathrm{~K}$ for $x=0.6,0.8,0.9$, and 0.95 are shown in Fig. 11 . The $\mathrm{U}$ contribution $\Delta \rho$ to the 


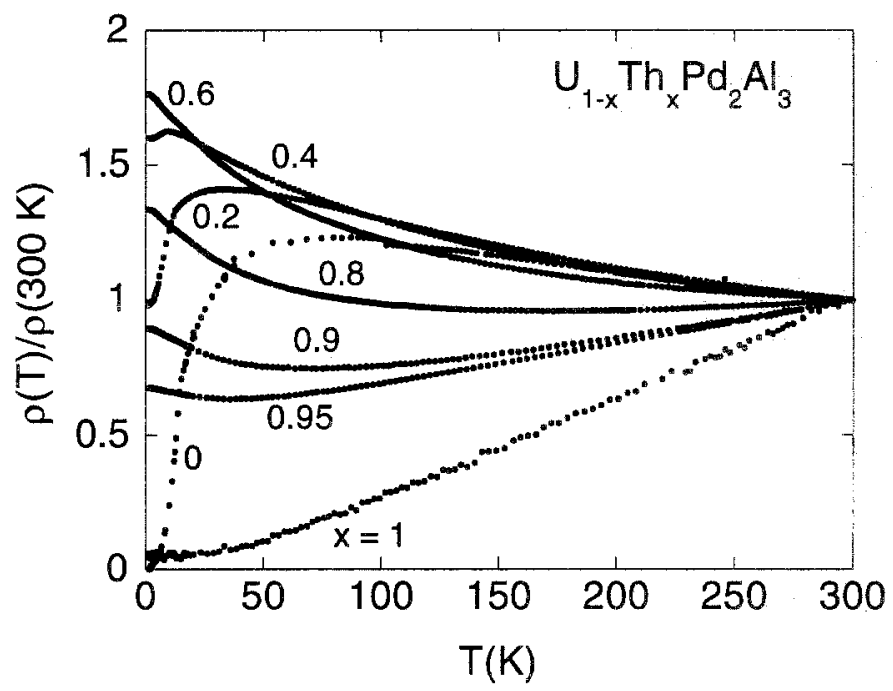

Fig. 10. Electrical resistivity $\rho(T)$, normalized to its room temperature value $\rho(300 \mathrm{~K})$, versus temperature $T$ for the $\mathrm{U}_{1-x} \mathrm{Th}_{x} \mathrm{Pd}_{2} \mathrm{Al}_{3}$ system for various values of $x$ between 0 and 1 .

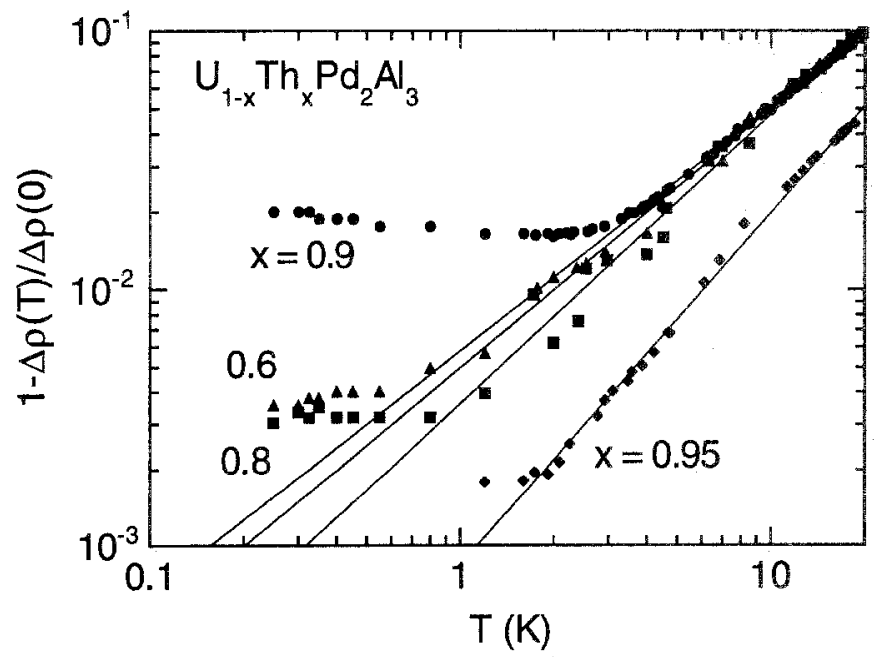

Fig. 11. Low temperature electrical resistivity data $(T \leq 20 \mathrm{~K})$ plotted as $\log (1-\Delta \rho(T) / \Delta \rho(0))$ versus $\log T$. Solid lines represent fits to a power law $\Delta \rho(T) / \Delta \rho(0)=1-a\left(T / T_{K}\right)^{n}$, with $n \approx 1$, indicative of NFL behavior. 


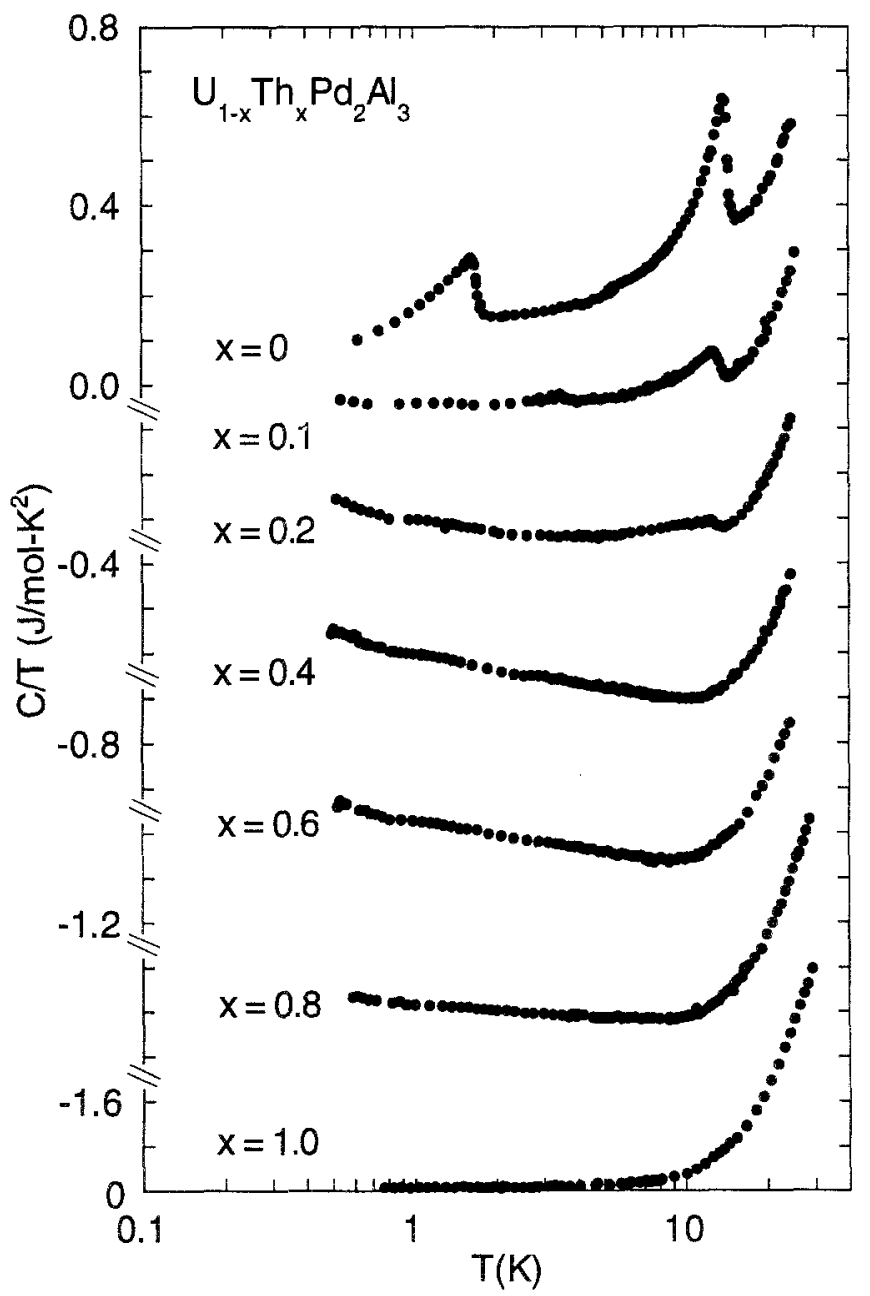

Fig. 12. Specific heat divided by temperature $C / T$ versus $T$ of $\mathrm{U}_{1-x} \mathrm{Th}_{x} \mathrm{Pd}_{2} \mathrm{Al}_{3}$ for various values of $x$ between 0 and 1 . 


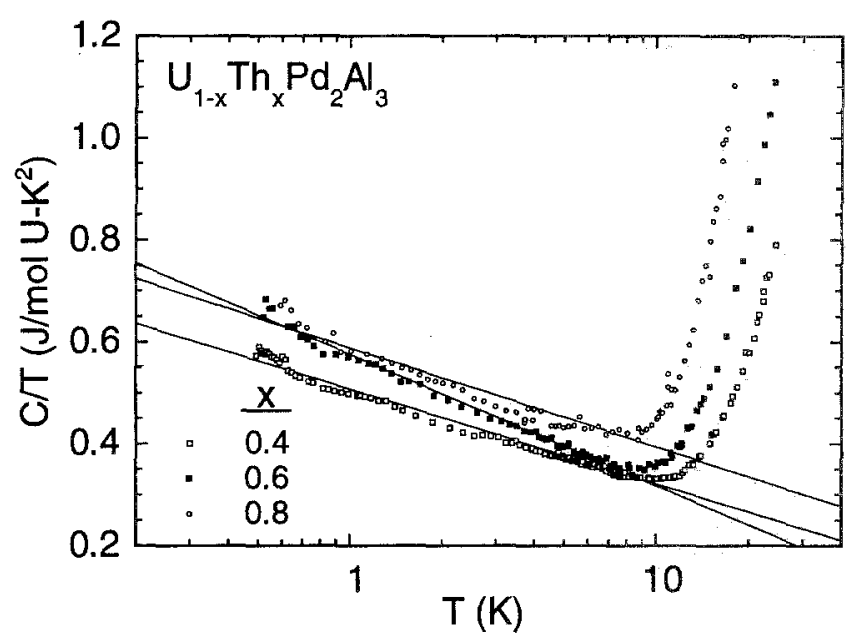

Fig. 13. Low temperature specific heat divided by temperature, $\mathrm{C} / \mathrm{T}$, versus $\log T$ for $\mathrm{U}_{1-x} \mathrm{Th}_{x} \mathrm{Pd}_{2} \mathrm{Al}_{3}$ alloys with $x=0.4,0.6$, and 0.8 .

resistivity was estimated by subtracting the resistivity of $\mathrm{ThPd}_{2} \mathrm{Al}_{3}$ from that of each $\mathrm{U}_{1-x} \mathrm{Th}_{x} \mathrm{Pd}_{2} \mathrm{Al}_{3}$ alloy to remove the phonon background contribution, at least to a first approximation. In the range $4 \mathrm{~K} \leq T \leq 20 \mathrm{~K}$, the data in Fig. 11 can be described by the relation $\Delta \rho(T)=\Delta \rho(0)\left(1-a\left(T / T_{K}\right)^{n}\right)$, yielding values for the residual resistivity $\Delta \rho(0)$, the slope $n$, and the intercept $a / T_{K}$. Taking $a=0.1$ to achieve agreement with the $T_{K}$ values calculated from the measured heat capacity, the fits yield the following values of $n$ and $T_{K}: n=1.0,1.1,0.95,1.4 ; T_{K}=22 \mathrm{~K}$, $21 \mathrm{~K}, 22 \mathrm{~K}, 35 \mathrm{~K}$; for $x=0.6,0.8,0.9,0.95$, respectively.

Displayed in Fig. 12 are plots of $C / T$ versus $\log T$ for various values of $x$ between 0 and 1 for $\mathrm{U}_{1-x} \mathrm{Th}_{x} \mathrm{Pd}_{2} \mathrm{Al}_{3}$. The specific heat anomalies due to $\mathrm{AFM}$ at $T_{N}$ and superconductivity at $T_{c}$, which are evident in the data for $x=0$, are rapidly suppressed with increasing $x$, similar to that observed in the $\mathrm{U}_{1-x} \mathrm{Th}_{x} \mathrm{Ru}_{2} \mathrm{Si}_{2}$ system. ${ }^{28}$ A $\ln T$ divergence in the $C / T$ data, emerging in the sample with $x=0.2$, is fully developed for the samples with $x=0.4,0.6$, and 0.8 . Plots of $\mathrm{C}(\mathrm{T}) / \mathrm{T}$, per mole $\mathrm{U} \mathrm{K}^{2}$, versus $\log \mathrm{T}$ are presented in Fig. 13 for the samples with $x=0.4,0.6$, and 0.8 . Analysis of the specific heat data in terms of the two-channel spin $1 / 2$ Kondo formula yields values of $T_{K}$ of $26 \mathrm{~K}, 18 \mathrm{~K}$, and $21 \mathrm{~K}$, for $x=0.4,0.6$, and 0.8 , respectively, in good agreement with the values inferred from the electrical resistivity. Experiments are in progress to determine whether the specific heat exhibits a sharp upturn below $\sim 0.4 \mathrm{~K}$, as it does in the $\mathrm{Y}_{1-x} \mathrm{U}_{x} \mathrm{Pd}_{3}$ system.

Figure 14 contains plots of the magnetic susceptibility $\chi$ versus temperature for the $\mathrm{U}_{1-x} \mathrm{Th}_{x} \mathrm{Pd}_{2} \mathrm{Al}_{3}$ system for various values of $x$ in the range $0 \leq x \leq 1$. The $\chi(T)$ data for $\mathrm{UPd}_{2} \mathrm{Al}_{3}$ exhibit a maximum near $40 \mathrm{~K}$ and an abrupt drop at $T_{N}=$ $14.5 \mathrm{~K}$. With increasing $x$, the maximum in $\chi(T)$ is suppressed until it disappears 


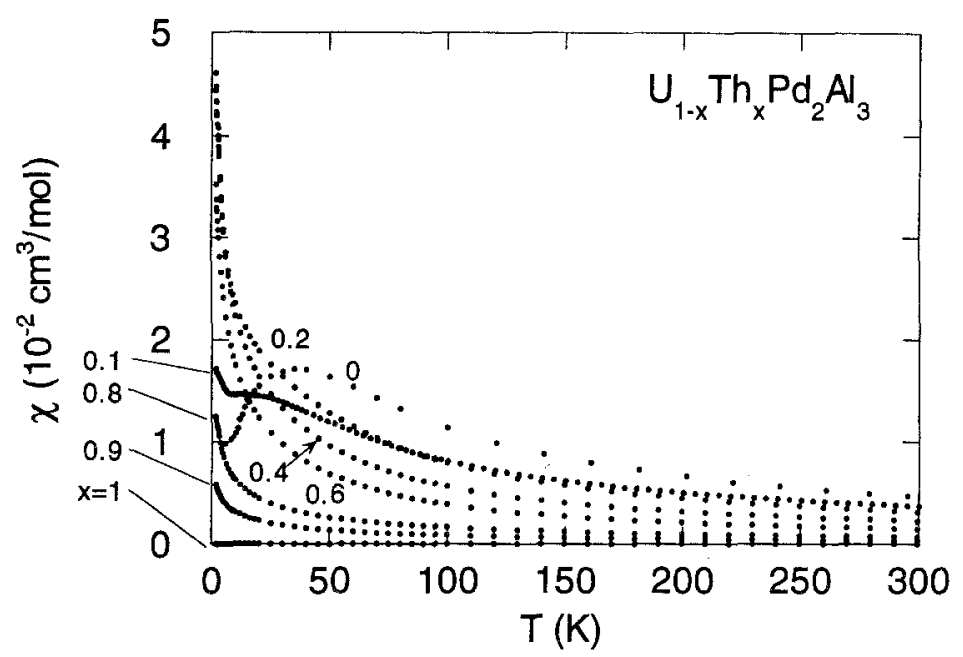

Fig. 14. Magnetic susceptibility $\chi$ versus temperature $\mathrm{T}$ for the $\mathrm{U}_{1-x} \mathrm{Th}_{x} \mathrm{Pd}_{2} \mathrm{Al}_{3}$ system for various values of $x$ between 0 and 1 .

at $x=0.1$ and, below $10 \mathrm{~K}$, is accompanied by an increase in $\chi$ with decreasing temperature. For values of $x>0.2, \chi(T)$ follows a Curie-Weiss law between $\sim$ $50 \mathrm{~K}$ and $300 \mathrm{~K}$; the effective magnetic moment $\mu_{\text {eff }}$ and Curie-Weiss temperature $\theta_{p}$ vary somewhat with $x$ and have values $\mu_{e f f} \approx 3.4 \mu_{B}$ and $\theta_{p} \approx-40 \mathrm{~K}$ in the NFL regime $x \geq 0.8$. Since for Kondo systems, $\left|\theta_{p}\right| \approx 3-4 T_{K}$, this suggests a value $T_{K} \approx 10 \mathrm{~K}$, in reasonable agreement with values of $T_{K}$ obtained from the scaling of the electrical resistivity and specific heat. At lower temperatures, the $\chi(T)$ data can be described by the relation $\chi(T)=\chi(0)\left(1-c\left(T / T_{K}\right)^{1 / 2}\right)$, with values of $T_{K}$ in reasonable agreement with those obtained from $\rho(T)$ and $C(T)$ for $c=1.15$. The values of $T_{K}$ are plotted in the low temperature phase diagram for $\mathrm{U}_{1-x} \mathrm{Th}_{x} \mathrm{Pd}_{2} \mathrm{Al}_{3}$ (Fig. 9), along with the values determined from $\rho(T)$ and $C(T)$. We have also compared the $\chi(T)$ data to the two-channel spin $1 / 2$ Kondo model result $\chi(T) \sim\left(-1 / T_{K}\right) \ln \left(T / c T_{K}\right)$ where $c=2.2\left(T \ll T_{K}\right)$. However, the fits are only qualitatively satisfactory and give values of $T_{K}$ that have a different dependence on $x$ than those obtained from $\rho(T)$ and $C(T)$.

\section{THE $\mathrm{UCu}_{5-x} \mathbf{P d}_{x}$ SYSTEM}

The family of compounds $\mathrm{UCu}_{5-x} \mathrm{Pd}_{x}$ is unique among the uranium based intermetallics known to have NFL scaling properties as it is a concentrated moment system. All $x \lesssim 2.5$ share the common $\mathrm{AuBe}_{5}$ structure, characterized by a periodic uranium lattice with two inequivalent copper sites. ${ }^{29}$ The parent compound $\mathrm{UCu}_{5}$ is a Kondo lattice antiferromagnet with Neél temperature $\mathrm{T}_{N}=15 \mathrm{~K}^{30}$ and a Kondo temperature of $\sim 10^{2} \mathrm{~K}$, taken from the quasielastic linewidth in inelastic neutron scattering experiments. ${ }^{31}$ With Pd doping $x$, the AFM is quickly suppressed, van- 


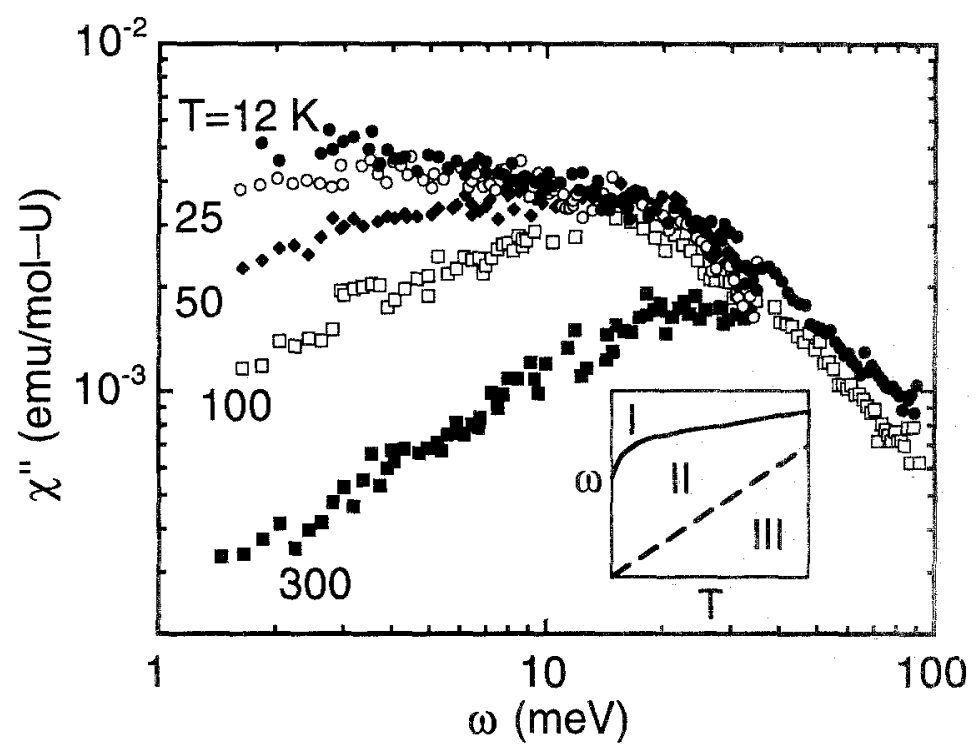

Fig. 15. $\chi^{\prime \prime}(\omega)$ for $\mathrm{UCu}_{4} \mathrm{Pd}$ at constant temperatures ranging from $12 \mathrm{~K}$ to $300 \mathrm{~K}$. Inset: three qualitatively different regimes are observed in the response: I. $\omega \geq \omega^{*}$ : $\chi^{\prime \prime}(\omega, T) \sim\left(\omega / T_{0}\right) /\left[1+\left(\omega / T_{0}\right)^{2}\right]$. II. $\omega \ll T: \chi^{\prime \prime}(\omega, T) \sim \omega / T^{\eta}, \eta=1 \pm 1 / 3$. III. $\omega \gg T: \chi^{\prime \prime}(\omega, T) \sim \omega^{-1 / 3}$. Solid line: $\omega^{*}(T)$, dashed line: $\omega=T$. From Ref 33

ishing for $x$ between 0.5 and $1 .^{29,32} \mathrm{~A}$ spin glass regime is observed for $x \geq 2$. The intermediate values $x=1,1.5$ display no long range order of any kind at the lowest temperatures, although thermal and transport measurements at temperatures below $\sim 20 \mathrm{~K}$ reveal that both have remarkable temperature and magnetic field scaling. ${ }^{2,32}$ These NFL properties are similar to those found in the dilute moment systems like $\mathrm{Y}_{0.8} \mathrm{U}_{0.2} \mathrm{Pd}_{3}$ described above, with the electrical resistivity $\rho(\mathrm{T})$ $\sim 1-a\left(T / T_{0}\right)$, the specific heat $C(T) / T \sim\left(-1 / T_{0}\right) \ln \left(T / b T_{0}\right)$, and static magnetic susceptibility $\chi(T) \sim 1-c\left(T / T_{0}\right)^{1 / 2}$ for both $\mathrm{UCu}_{3.5} \mathrm{Pd}_{1.5}$ and $\mathrm{UCu}_{4} \mathrm{Pd}$. These weak low temperature divergences suggest the existence of magnetic excitations with unusual scaling properties.

We have used neutron time of flight measurements ${ }^{33}$ performed for energy. transfers between $0.5 \mathrm{meV}$ and $400 \mathrm{meV}$ at temperatures from $10 \mathrm{~K}$ to $300 \mathrm{~K}$ to study the frequency/temperature scaling properties of these excitations. Although both systems display virtually identical temperature dependences in $p, C$, and $\chi$, the nature of the magnetic excitations for $\mathrm{UCu}_{3.5} \mathrm{Pd}_{1,5}$ and $\mathrm{UCu}_{4} \mathrm{Pd}$ are qualitatively different from those of $\mathrm{Y}_{0.8} \mathrm{U}_{0.2} \mathrm{Pd}_{3} \cdot{ }^{18,34}$ The uranium ions in the latter compound have a nonmagnetic ground state, and a magnetic response $S(\omega)$ consisting of two inelastic crystal field levels at $\sim 5 \mathrm{meV}$ and $\sim 16 \mathrm{meV}$. In contrast, we have found a broad, quasielastic magnetic response $\mathrm{S}(\omega)$ which is virtually identical for $\mathrm{UCu}_{4} \mathrm{Pd}$ and $\mathrm{UCu}_{3.5} \mathrm{Pd}_{1.5}$, indicative of a magnetic ground state for the uranium ions, with 


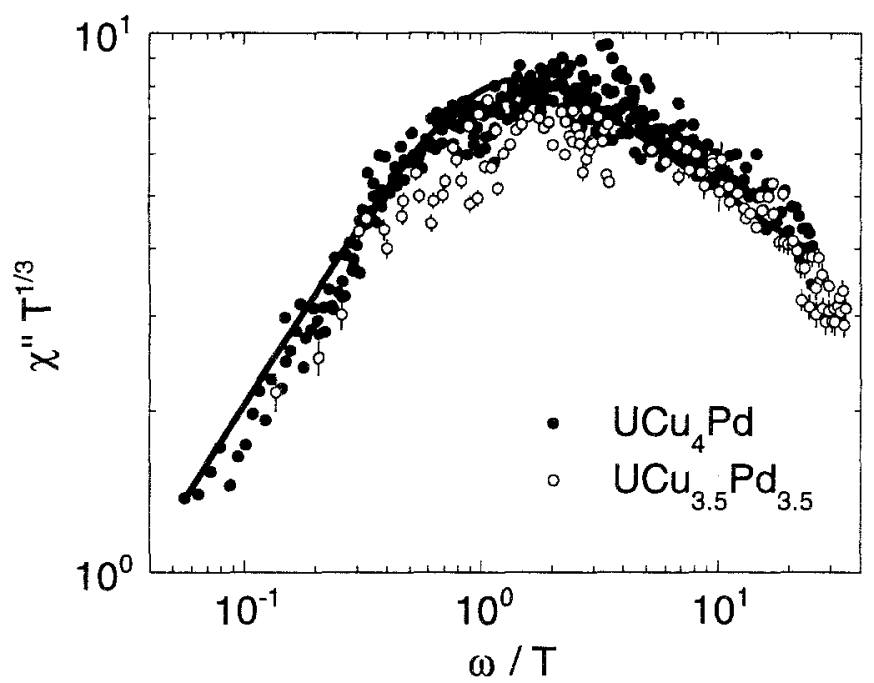

Fig. 16. $\chi^{\prime \prime}(\omega, T) T^{1 / 3}$ has almost identical universal scaling properties for $\mathrm{UCu}_{4} \mathrm{Pd}$ (filled circles) and $\mathrm{UCu}_{3.5} \mathrm{Pd}_{1.5}$ (open circles). Data with energy transfers $\omega \geq 25 \mathrm{meV}$ are not included in this plot. Solid line: $\chi^{\prime \prime}(\omega, T) T^{1 / 3}$ $\sim(T / \omega)^{\overline{1} / 3} \tanh (\omega / 1.2 T)$. From Ref. ${ }^{33}$

no evidence for distinct crystal field excitations. Further, $S(\omega)$ displays no appreciable wavevector dependence beyond that of the $\mathrm{U}^{3+} / \mathrm{U}^{4+}$ magnetic form factor so in every case we have summed the magnetic response over experimental wavevectors.

The detailed frequency and temperature dependences of the dynamical susceptibilities $\chi(\omega, T)$ of $\mathrm{UCu}_{5-x} \operatorname{Pd}_{x}(x=1,1.5)$ are obtained from $S(\omega, T)$ by

$$
S(\omega)=(n(\omega)+1) \chi^{\prime \prime}(\omega, T)
$$

where $n(\omega)+1$ is the thermal occupation factor. The resulting $\chi^{\prime \prime}(\omega, T)$ is detailed for $\mathrm{UCu}_{4} \mathrm{Pd}$ in Fig. 15. As schematically indicated in the inset, there are three qualitatively different regimes of behavior. At every temperature, a quasi-Lorentzian regime is found for energy transfers $\omega \geq \omega^{*} \cong 25 \mathrm{meV}$. Here, $\chi^{\prime \prime}(\omega, T)=\chi_{\text {Lor }}(\omega / \Gamma) /\left(1+(\omega / \Gamma)^{2}\right)$ has a weak temperature dependence stemming from that of $\chi_{L o r}$ and $\Gamma$. For $\omega<\omega^{*}, \chi^{\prime \prime}(\omega, T)$ is well described by a universal scaling relation

$$
\chi^{\prime \prime}(\omega, T) T^{1 / 3}=(T / \omega)^{1 / 3} \mathcal{Z}(\omega / T)
$$

demonstrated in Fig. 16 for both $\mathrm{UCu}_{4} \mathrm{Pd}$ and $\mathrm{UCu}_{3.5} \mathrm{Pd}_{1.5}$. While our choice may not be unique, it is clear from Fig. 16 that $\mathcal{Z} \sim \tanh (\omega / 1.2 T)$ fits the data very well. $\mathcal{Z} \sim 1 /(\mathrm{n}(\omega)+1)$ is another intriguing possibility, although this scaling function fails for $T>\omega^{*}$.

The scaling behavior found for $\omega<\omega^{*}$ represents a qualitatively new type of magnetic response for an f-electron based system. It is interesting to investigate 


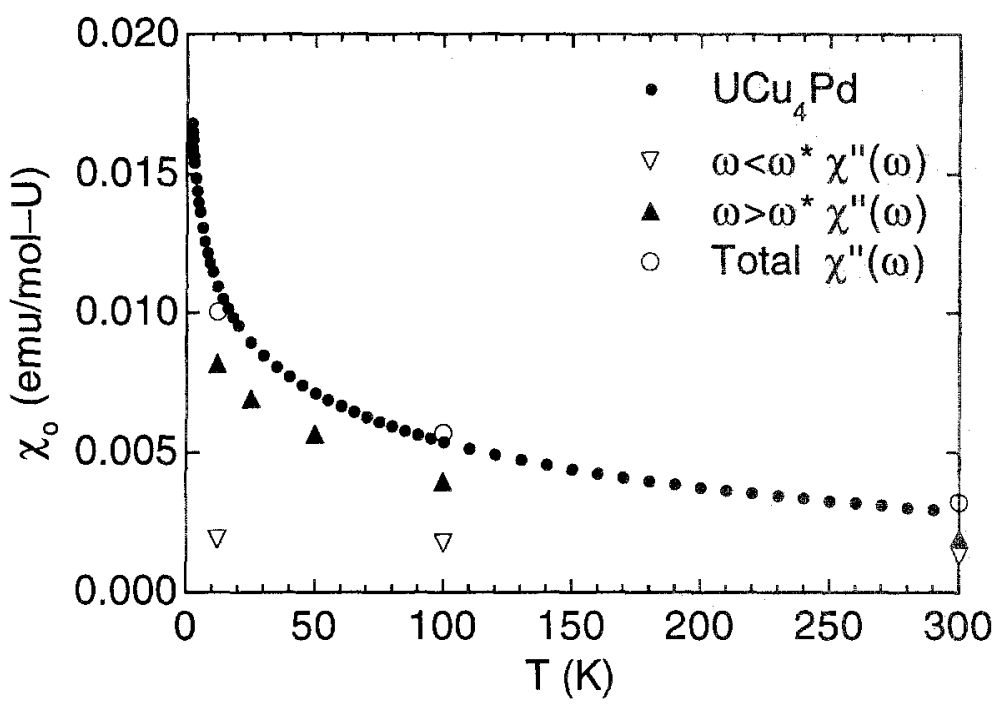

Fig. 17. A Comparison of the measured static susceptibility $\chi(T)$ to that calculated from the measured dynamical susceptibility (see text) for energy transfers larger (filled triangles) and smaller (open triangles) than $\omega^{*}=25 \mathrm{meV}$. Integrating over the total response for $1 \mathrm{meV} \leq \omega \leq 200 \mathrm{meV}$ (open circles) accounts for virtually the entire measured $\chi(T)$.

how these unusual excitations are reflected in the temperature dependences of static properties, in particular, the static susceptibility $\chi(T)$. A Kramers-Kronig relation links $\chi^{\prime \prime}(\omega, T)$ and $\chi(T)$ :

$$
\chi(T)=\frac{2}{\pi} \int_{0}^{\infty} d \omega \frac{\chi^{\prime \prime}(\omega, T)}{\omega}=\int_{0}^{\omega^{*}} d \omega \frac{\omega^{-1 / 3} \mathcal{Z}(\omega / T)}{\omega}+\int_{\omega^{*}}^{\infty} d \omega \frac{\chi_{\operatorname{Lor} \omega / \Gamma}}{\omega\left(1+(\omega / \Gamma)^{2}\right)} .
$$

That is, both the NFL like excitations for $\omega<\omega^{*}$ and the higher energy localized magnetic excitations contribute, in principle, to the temperature dependence of the measured $\chi(T)$. We have performed numerical integrations over both of the terms appearing in Eq. (7) to determine the relative magnitudes of the two contributions in $\mathrm{UCu}_{4} \mathrm{Pd}$, depicted in Fig. 17.

The high energy, localized moment excitations $\left(\omega>\omega^{*}\right)$ make a Curie-Weiss like contribution to $\chi_{0}$, which is almost temperature independent for $T<300 \mathrm{~K}$. The temperature dependence of $\chi(T)$ is consequently dominated for $85 \mathrm{~K} \lesssim T \lesssim$ $300 \mathrm{~K}$ by the low energy excitations $\left(\omega<\omega^{*}\right)$, and reflects their unusual scaling properties. As demonstrated in Fig. 17, the sum of these two terms agrees very well with the measured static susceptibility $\chi(T)$, indicating that relatively little of the magnetic response falls outside our experimental energy window, $1 \mathrm{meV} \leq \omega \leq$ 
$200 \mathrm{meV}$. However, extending this window to lower energy transfers is imperative to establish whether the non Fermi liquid scaling properties of the excitations we have observed correspond to true critical scattering related to a $T=0 \mathrm{~K}$ phase transition or, alternatively, represent an intermediate temperature/frequency crossover regime preceding the actual critical regime.

\section{CONCLUDING REMARKS}

It is apparent that NFL behavior in f-electron systems is a general phenomenon. Low temperature NFL characteristics have been identified in the physical properties of nearly ten f-electron systems. With a few possible exceptions (e.g., $\mathrm{UBe}_{13}{ }^{35}$ ), all of the examples of NFL behavior in f-electron systems are found in alloy systems. The main result of the alloying process seems to be to "tune" the system into the NFL regime, although the alloying also introduces disorder into the system, which may also play an important role. The NFL behavior is found in two types of systems: (1) Systems containing small concentrations of rare earth and actinide ions with partially-filled f-electron shells, where the NFL behavior appears to be a single ion effect. Examples of this type of system include $\mathrm{M}_{1-x} \mathrm{U}_{x} \mathrm{Pd}_{3}(\mathrm{M}=\mathrm{Sc}, \mathrm{Y})$, $\mathrm{Th}_{1-x} \mathrm{U}_{x} \mathrm{Ru}_{2} \mathrm{Si}_{2},{ }^{36} \mathrm{La}_{0.9} \mathrm{Ce}_{0.1} \mathrm{Cu}_{2} \mathrm{Si}_{2},{ }^{37}$ and $\mathrm{U}_{1-x} \mathrm{Th}_{x} \mathrm{Pd}_{2} \mathrm{Al}_{3}$ for small $\mathrm{U}$ concentrations. (2) Systems containing large concentrations of rare earth or actinide ions with partially-filled f-electron shells, which sometimes form an ordered sublattice, where the NFL behavior appears to due to interactions between the ions. Examples of this type of system include $\mathrm{CeCu}_{5.9} \mathrm{Au}_{0.1}{ }^{38}$ and $\mathrm{UCu}_{3.5} \mathrm{Pd}_{1.5}{ }^{32}$ In the $\mathrm{CeCu}_{5-x} \mathrm{Au}_{x}$ system, the NFL regime exists in a narrow range of $x$-values between a nonmagnetic heavy fermion ground state at $x=0$ and an antiferromagnetically ordered state at $x=0.2$.

Many of the f-electron systems in which NFL behavior is found exhibit the Kondo effect as evidenced by an increase of the electrical resistivity with decreasing temperature and a magnetic susceptibilitiy $\chi$ at high temperatures $\left(T \gg T_{K}\right)$ that follows a Curie-Weiss law with a negative Curie-Weiss temperature $\left|\theta_{p}\right| \approx\left(3-4 T_{K}\right)$. In the cases where $T_{K}$ can be estimated from the $\ln T$ behavior of the electrical resistivity or the Curie-Weiss temperature, the NFL behavior occurs at temperatures much less than $T_{K}$. The NFL behavior is manifested in the temperature dependences of the electrical resistivity, specific heat, and magnetic susceptibility for $T \ll T_{K}: \rho(T) \sim 1-a T / T_{K}$ where $a$ can be $>0$ (usual Kondo effect) or $<0$, $C(T) / T \sim\left(-1 / T_{K}\right) \ln \left(T / b T_{K}\right)$, and $\chi(T) \sim 1-c\left(T / T_{K}\right)^{1 / 2}$, where $|a|, b$, and $c$ are constants of the order of unity. These low temperature properties scale with $T_{K}$ where $T_{K}$ is comparable to the value determined from the high temperature Kondo behavior when $a>0$. This is summarized in Table 2, which is an updated version of a table we first presented in reference 2 . However, these temperature dependences of $\rho, C$, and $\chi$ have also been observed in f-electron systems in which a Kondo effect has not been clearly identified. Therefore, we refer to a characteristic temperature $T_{0}$ that is equal to $T_{K}$ in the systems where the Kondo effect is operative. The two new entries are for $\mathrm{M}_{0.1} \mathrm{U}_{0.9} \mathrm{Ni}_{2} \mathrm{Al}_{3}{ }^{39}\left(\mathrm{M}=\mathrm{Pr}\right.$, Th) and $\mathrm{Ce}_{1-x} \mathrm{Th}_{x} \mathrm{RhSb}^{40}$ Materials conforming to these temperature dependences are indicated by "yes" and the exceptions by an entry describing the behavior, or a "dash" where the behavior has not yet been established. As we stated in reference 2, taken of face value, 
Table 2. Examples of f-electron systems which exhibit characteristic non Fermi liquid behavior in the low temperature electrical resistivity $\rho(T) \sim 1-a T / T_{0}$, specific heat $C(T) / T \sim\left(-1 / T_{0}\right) \ln \left(T / b T_{0}\right)$, and magnetic susceptibility $\chi(T) \sim$ $1-c\left(T / T_{0}\right)^{1 / 2}$.

\begin{tabular}{lcccc}
\hline System & $\rho$ & $C / T$ & $\chi$ & $T_{0}^{*}(K)$ \\
\hline $\mathrm{La}_{0.9} \mathrm{Ce}_{0.1} \mathrm{Cu}_{2} \mathrm{Si}_{2}$ & yes & yes & - & 9 \\
$\mathrm{M}_{1-x} \mathrm{U}_{x} \mathrm{Pd}_{3}(\mathrm{M}=\mathrm{Sc}, \mathrm{Y})$ & yes & yes & yes & $40-220$ \\
$\mathrm{UCu}_{3.5} \mathrm{Pd}_{1.5}$ & yes & yes & yes & 28 \\
$\mathrm{U}_{1-x} \mathrm{Th}_{x} \mathrm{Pd}_{2} \mathrm{Al}_{3}$ & yes & yes & yes & 20 \\
$\mathrm{M}_{0.1} \mathrm{U}_{0.9} \mathrm{Ni}_{2} \mathrm{Al}_{3}(\mathrm{M}=\mathrm{Pr}, \mathrm{Th})$ & - & yes & yes & 200 \\
$\mathrm{Ce}_{1-x} \mathrm{Th}_{x} \mathrm{RhSb}$ & - & yes & - & 33 \\
$\mathrm{Th}_{1-x} \mathrm{U}_{x} \mathrm{Ru}_{2} \mathrm{Si}_{2}$ & $(\mathrm{a}<0)$ & yes & $-\ln \left(T / b T_{K}\right)$ & 11 \\
$\mathrm{U}_{0.9} \mathrm{Th}_{0.1} \mathrm{Be}_{13}$ & $(a<0)$ & yes & yes & 8 \\
$\mathrm{CeCu}_{5.9} \mathrm{Au}_{0.1}$ & $(\mathrm{a}<0)$ & yes & yes & 3.5 \\
\hline
\end{tabular}

* Deduced from the slope $A=d(C / T) / d \ln T$ of the logarithmic divergence in the specific heat: $T_{0}=-0.251 R / A$

the results presented in Table 2 suggest that the low temperature behavior of the electrical resistivity, specific heat, and magnetic susceptibility of f-electron systems that exhibit NFL behavior have some common characteristics, irrespective of the microscopic mechanisms responsible for the NFL behavior.

Since many examples of NFL behavior are found in f-electron systems that exhibit the Kondo effect, the multichannel Kondo model would be expected to provide an explanation since it predicts that the physical properties have NFL characteristics at low temperatures $T \ll T_{K}$. The two-channel spin $1 / 2$ Kondo model does, in fact, give $C(T) / T \sim\left(-1 / T_{K}\right) \ln \left(T / b T_{K}\right)$, which agrees with experiment, but predicts that $\rho(T) \sim 1-a\left(T / T_{K}\right)^{1 / 2}$ and $\chi(T) \sim\left(-1 / T_{K}\right) \ln \left(T / c T_{K}\right)$, in disagreement with experiment. The quadrupolar Kondo model, which maps onto the two channel spin $1 / 2$ Kondo model, can account for the $1-c\left(T / T_{K}\right)^{1 / 2}$ dependence of the magnetic susceptibility at low temperature. For a magnetic two channel spin $1 / 2$ Kondo effect, the magnetic susceptibility is also found to diverge logarithmically at $T=0$, while for a two channel quadrupolar Kondo effect, it is the electric quadrupolar susceptibility that diverges logarithmically. The quadrupolar susceptibility can be extracted from ultrasound measurements. For the quadrupolar Kondo effect, the magnetic susceptibility is van Vleck in character, resulting from coupling of the $\Gamma_{3}$ ground state to the $\Gamma_{5}$ and $\Gamma_{4}$ magnetic excited states. ${ }^{35}$ Cox and Makivic have estimated that $\chi(T)$ should vary like $\chi(T)=\chi(0)\left(1-c\left(T / T_{K}\right)^{1 / 2}\right)$ at low temperatures, where $\chi(0)$ is of the order of the van Vleck susceptibility and the coefficient $c \approx 1 .^{41}$ As we have discussed in Section 2 , this seems to be the case for $\mathrm{Y}_{1-x} \mathrm{U}_{x} \mathrm{Pd}_{3}$ where the ground state of the $\mathrm{U}^{4+}$ ions in the cubic $\mathrm{CEF}$ appears to be a $\Gamma_{3}$ nonmagnetic doublet. However, a quadrupolar Kondo effect would not be expected to be appropriate for all of the other f-electron systems which exhibit NFL behavior. Furthermore, the quadrupolar Kondo model cannot account for the linear temperature dependence of the electrical resistivity. One system that does appear to satisfy the predictions of the quadrupolar Kondo model for $\rho(T), C(T)$, and $\chi(T)$, is $\mathrm{U}_{0.9} \mathrm{Th}_{0.1} \mathrm{Be}_{13}$, according to recent measurements by Aliev et al. ${ }^{42}$ 
although in this case the coefficient $a$ that appears in the resistivity is negative. It will be interesting to see whether $U$ is tetravalent in $U_{0.9} T_{0.1} \mathrm{Be}_{13}$ and has a nonmagnetic $\Gamma_{3}$ doublet ground state in the cubic CEF.

Andraka and Tsvelik ${ }^{43}$ have raised several objections to the multichannel Kondo interpretation of the NFL behavior in $\mathrm{Y}_{1-x} \mathrm{U}_{x} \mathrm{Pd}_{3}$ and other $\mathrm{f}$-electron systems. One of these objections is based on specific heat measurements on $\mathrm{Y}_{0.8} \mathrm{U}_{0.2} \mathrm{Pd}_{3}$ where they find that the specific heat scales as $C(H, T) / T$ $C(0, T) / T=-f\left(H / T^{\beta}\right)$ with $\beta=1.3 \pm 0.1$. They argue that a scaling dimension $\beta$ greater than one precludes a single site interpretation of this phenomenon. Moreover, it has been noted ${ }^{44}$ that scaling of the magnetic field for a two channel spin $1 / 2$ Kondo effect has predicted scaling dimensions of $1 / 2$ for the magnetic case ${ }^{12}$ and $1 / 4$ for the nonmagnetic (quadrupolar) case, ${ }^{45}$ well outside the experimental uncertainty of $\beta$. A phenomenological theory for the NFL behavior of f-electron systems such as $\mathrm{Y}_{0.8} \mathrm{U}_{0.2} \mathrm{Pd}_{3}$ and $\mathrm{UCu}_{3.5} \mathrm{Pd}_{1.5}$ has been proposed by Tsvelik and Reizer. ${ }^{46}$ According to this theory, the alloys have a critical point at $T=0 \mathrm{~K}$ so that their low temperature thermodynamics is determined by collective modes corresponding to fluctuations in the order parameter in the vicinity of a critical point, rather than single particle fermion excitations, as in a Fermi liquid. This scenario would seem to be applicable to the $\mathrm{CeCu}_{5.9} \mathrm{Au}_{0.1}$ system, mentioned above. However, we caution that the scaling exponents deduced from experiment so far lack internal consistency within the context of this particular theory. Nonetheless, the NFL behavior in systems such as $\mathrm{Y}_{1-x} \mathrm{U}_{x} \mathrm{Pd}_{3}$ and $\mathrm{U}_{1-x} \mathrm{Th}_{x} \mathrm{Pd}_{2} \mathrm{Al}_{3}$, which definitely do exhibit Kondo like features in $\rho(T)$ and $\chi(T)$ at high temperatures $T \lesssim T_{K}$, persists into the dilute $U$ concentration limit, suggesting that a single site mechanism such as a multichannel Kondo effect is operative.

\section{ACKNOWLEDGMENTS}

We thank our many collaborators for their contributions to this work. Research at UCSD was supported by the U.S. NSF under Grant No. DMR-9107698 and the U.S. DOE under Grant No. DE-FG03-86ER45230. Some equipment used in this research was provided by the Center for Interface and Materials Science and funded by the W. A. Keck Foundation. Work at Los Alamos and Argonne was carried out under the auspices of the U.S. DOE, Office of Basic Energy Sciences, the latter under Contract No. W-31-109-ENG-38. J. H. acknowledges financial support from the German Academic Exchange Service. M. C. de A. acknowledges financial support from CNPq, Brazil. M.C.A. is grateful to the Phoenix Memorial Project, University of Michigan, for travel support.

\section{REFERENCES}

1. C. L. Seaman, M. B. Maple, B. W. Lee, S. Ghamaty, M. S. Torikachvili, J.-S. Kang, L. Z. Liu, J. W. Allen, and D. L. Cox, Phys, Rev. Lett. 67, 2882 (1991); J. Alloys and Compounds 181, 327 (1992).

2. M. B. Maple, C. L. Seaman, D. A. Gajewski, Y. Dalichaouch, V. B. Barbetta, M. C. de Andrade, H. A. Mook, H. G. Lukefahr, O. O. Bernal, and D. E. MacLaughlin, J. Low Temp. Phys. 95, 225 (1994). 
3. K. A. McEwen, U. Steigenberger, and J. L. Martinez, Physica B 186-188, 670 (1992).

4. W. D. Wu, A. Keren, L. P. Le, G. M. Luke, Y. J. Uemura, C. L. Seaman, Y. Dalichaouch, and M. B. Maple, Physica B 186-188, 344 (1993); Phys. Rev. Lett. 72, 3722 (1994).

5. J.-S. Kang, J. W. Allen, M. B. Maple, M. S. Torikachvili, W. P. Ellis, B. B. Pate, Z.-X. Shen, J. J. Yeh, and I. Lindau, Phys. Rev. B 39, 13529 (1989).

6. C. Koenig, Z. Phys. B 50, 33 (1983).

7. M. J. Besnus, J. P. Kappler, and A. Meyer, J. Phys. F: Met. Phys. 13, 597 (1983).

8. C. L. Seaman and M. B. Maple, Physica B 199-200, 396 (1994).

9. J. W. Allen, L. Z. Liu, R. O. Anderson, C. L. Seaman, M. B. Maple, Y. Dalichaouch, J.-S. Kang, M. S. Torikachvili, and M. A. Lòpez de la Torre, Physica B 186-188, 307 (1993).

10. D. A. Gajewski, P. Allenspach, C. L. Seaman, and M. B. Maple, Physica B 199-200, 419 (1994).

11. L. Degiorgi, H. R Ott, and F. Hulliger, preprint (1994).

12. A. M. Tsvelik, J. Phys. C 18, 159 (1985); P. D. Sacramento and P. Schlottmann, Phys. Lett. A 142, 245 (1989).

13. H.R. Ott, E. Felder, and A. Bernasconi, Physica B 186-188, 207 (1993).

14. J. Mc Carten, S. E. Brown, C. L. Seaman, and M. B. Maple, Phys. Rev. B 49, $6400(1994)$.

15. M. B. Maple, D. A. Gajewski, C. L. Seaman, and J. W. Allen, Physica B 199. 200, 423 (1994).

16. P. D. Sacramento and P. Schlottmann, Phys. Rev. B 43, 13294 (1991).

17. K. R. Lea, M. J. M. Leask, and W. P. Wolf, J. Phys. Chem. Solids 23, 1381 (1962).

18. H. A. Mook, C. L. Seaman, M. B. Maple, M. A. Lòpez de la Torre, D. L. Cox, and M. Makivic, Physica B 186-188, 341 (1993).

19. M. Amara, D. Finsterbusch, B. Luy, B. Lüthi, F. Hulliger, and H. R. Ott, to be published.

20. C. Geibel, S. Thies, D. Kaczorowski, A. Mehner, A. Grauel, B. Seidel. U. Ahlheim, R. Helfrich, K. Peterson, C. D. Bredl, and F. Steglich, Z. Phys. $B$ 83, 305 (1991).

21. C. Geibel, C. Schank, S. Thies, H. Kitazawa, C. D. Bredl, A. Bohm, M. Rau, A. Grauel, R. Caspary, R. Helfrich, U. Ahlheim, G. Weber, and F. Steglich, $Z$. Phys. B 84, 1 (1991).

22. A. Krimmel, P. Fischer, B. Roessli, H. Maletta, C. Geibel, C. Schank, A. Graul, A. Loidl, and F. Steglich, Z. Phys. B 86, 161 (1991).

23. C. Broholm, J. K. Kjems, W. J. L. Buyers, P. Matthews, T. T. M. Palstra, A. A. Menovsky, and J. A. Mydosh, Phys. Rev. Lett. 58, 1467 (1987).

24. G. Aeppli, E. Bucher, C. Broholm, J. K. Kjems, J. Baumann, and H. Hufnagl, Phys. Rev. Lett. 60, 615 (1985).

25. Y. Dalichaouch, M. C. de Andrade, and M. B. Maple, Phys. Rev. B 43, 299 (1992).

26. M. B. Maple, J. W. Chen, Y. Dalichaouch, T. Kohara, C. Rossel, M. S. Torikachvili, M. W. McElfresh, and J. D. Thompson, Phys. Rev. Lett. 56, 185 
(1986).

27. Y. Dalichaouch and M. B. Maple, Physica B 199 \& 200, 176 (1994).

28. M. A. Lopez de la Torre, P. Visani, Y. Dalichaouch, B. W. Lee, and M. B. Maple, Physica B 179, 208 (1992).

29. K. H. J. Buschow, A. S. van der Goot, and J. Birkhan, J. Less Comm. Metals 19, 433(1969); R. Troc, V. Tran, and Z. Zolnierek, J. Magn. Magn. Mater. $90-91,405(1990)$.

30. Z. Fisk, G. R. Stewart, J. O. Willis, H. R. Ott, and F. Hulliger, Phys. Rev.B 30, 6360(1984).

31. U. Walter, M. Loewenhaupt, E. Holland-Moritz, and W. Schlabitz, Phys. Rev. $B$ 36, 1981(1987).

32. B. Andraka and G. R. Stewart, Phys. Rev. B 47, 2886 (1993); B. Andraka, Physica B 199 - 200, 239 (1994).

33. M. C. Aronson, R. Osborn, R. A. Robinson, J. W. Lynn, R. Chau, C. L. Seaman, and M. B. Maple, to appear in Physica B (1994).

34. K. A. McEwen, M. J. Bull, and R. S. Eccleston, to appear in Physica B (1994).

35. D. L. Cox, Phys. Rev. Lett. 59, 1240 (1987).

36. H. Amitsuka, T. Hidano, T. Honmo, H. Mitamura, and T. Sakakihara, Physica B 186-188, 337 (1993).

37. B. Andraka, Phys. Rev. B 49, 3589 (1994).

38. H. v. Löhneysen, T. Pietrus, G. Portisch, H. G. Schlager, A. Schröder, M. Sieck, and T. Trappmann, Phys. Rev. Lett. 72, 3262 (1994).

39. B. Andraka, Phys. Rev. B 49, 348 (1994).

40. W. W. Kim, J. S.Kim, B. Andraka, and G. R. Stewart, Phys. Rev. B 47, 3589 (1993).

41. D. L. Cox and M. Makivic, to appear in Physica B (1994).

42. F. G. Aliev, S. Vieira, R. Villar, J. L. Martinez, C. L. Seaman, and M. B. Maple, to be published.

43. B. Andraka and A. M. Tsvelik, Phys. Rev. Lett. 67, 2886 (1991).

44. B. Andraka, Physica B 199 - 200, 239(1994).

45. A. M. Tsvelik, private communication in Ref. 43 .

46. A. M. Tsvelik and M. Reizer, Phys. Rev. B 48, 9887 (1993). 